\title{
Abelian extensions via prequantization
}

\author{
Cornelia Vizman \\ West University of Timisoara, Department of Mathematics \\ Bd. V.Parvan 4, 300223-Timisoara, Romania \\ e-mail:vizman@math.uvt.ro
}

\begin{abstract}
We generalize the prequantization central extension of a group of diffeomorphisms preserving a closed 2-form $\omega$, to an abelian extension of a group of diffeomorphisms preserving a closed vector valued 2-form $\omega$ up to a linear isomorphism ( $\omega$-equivariant diffeomorphisms). Every abelian extension of a simply connected Lie group can be obtained as the pull-back of such a prequantization abelian extension.
\end{abstract}

Keywords: prequantization, diffeomorphism group, flux 1-cocycle, abelian extension

$M S C: 22 \mathrm{E} 65,58 \mathrm{~B} 20$

\section{Introduction}

Not every infinite dimensional Lie algebra can be integrated to a Lie group. Moreover, given a Lie group $G$ with Lie algebra $\mathfrak{g}$, not every abelian Lie algebra extension of $\mathfrak{g}$ can be integrated to an abelian Lie group extension of $G$. The two obstructions to the integrability of such abelian extensions are described in terms of a flux homomorphism involving $\pi_{1}(G)$ and a period homomorphism involving $\pi_{2}(G)$ [10] (see the appendix).

Taking this into account, geometric constructions of Lie group extensions in infinite dimensions are important. A prototype is the prequantization extension [7] 17] associated to a prequantizable symplectic manifold $(M, \omega)$ :

$$
1 \rightarrow S^{1} \rightarrow \operatorname{Quant}(P, \theta)_{0} \rightarrow \operatorname{Ham}(M, \omega) \rightarrow 1 .
$$

Here $P$ is a principal circle bundle over $M$ with connection $\theta$ and curvature $\omega$. The identity component of the group of quantomorphisms, i.e. connection preserving automorphisms, is a 1-dimensional central extension of the group of hamiltonian diffeomorphisms of $M$. This is a Lie group extension when $M$ is compact [14]. 
Central Lie group extensions of a Lie group $G$ can be obtained as pull-back of the prequantization extension, given a hamiltonian $G$-action on the prequantizable symplectic manifold $(M, \omega)$.

This construction works more generally for closed vector valued 2-forms $\omega$ on a smoothly paracompact manifold $M$, possibly infinite dimensional, with values in a Mackey complete locally convex vector space $V$, and with discrete period group $\Gamma \subset V$, i.e. for prequantizable forms. The identity component of the "quantomorphism group" $\operatorname{Diffeq}^{\text {eq }}(P, \theta)^{A}$ of the principal bundle $q: P \rightarrow M$ with abelian structure group $A=V / \Gamma$ and principal $A$-action $\rho$ is an abelian extension of the "hamiltonian group" Diffex $(M, \omega)$

$$
1 \rightarrow A \rightarrow \operatorname{Diff}(P, \theta)_{0}^{A} \rightarrow \operatorname{Diff}_{\mathrm{ex}}(M, \omega) \rightarrow 1 .
$$

In the infinite dimensional setting, the prequantization extension is not a Lie group extension, but the pull-back provides Lie group extensions of Lie groups with hamiltonian actions $(M, \omega)$ [11. For instance, all the central extensions of the group of exact volume preserving diffeomorphisms can be obtained from its hamiltonian action on the non-linear Grassmannian of codimension two submanifolds of $M$ [5] [4].

Our main goal is to construct a prequantization abelian extension associated to a prequantizable form $\omega \in \Omega^{2}(M, V)$ :

$$
1 \rightarrow A \rightarrow \operatorname{Diff}^{\mathrm{eq}}(P, \theta)_{0}^{A} \rightarrow \operatorname{Diff}_{\mathrm{ex}}^{\mathrm{eq}}(M, \omega) \rightarrow 1 .
$$

The identity component of the group $\operatorname{Diffeq}^{e q}(P, \theta)^{A}$ of equi-quantomorphisms is an abelian extension of the group Diffeq $(M, \omega)$ of equi-hamiltonian diffeomorphisms. We show that, in the context of abelian extensions, it plays the same role as the prequantization extension in the context of central extensions. Moreover, Theorem 5.2 shows that every abelian Lie group extension of a simply connected Lie group can be obtained as pull-back of such a prequantization abelian extension.

An $\omega$-equivariant $G$-action involves an action on $M$ and one on $V$. The group

$$
\operatorname{Diffeq}^{e}(M, \omega):=\left\{(\varphi, u) \in \operatorname{Diff}(M) \times \operatorname{GL}(V): \varphi^{*} \omega=u \cdot \omega\right\}
$$

of $\omega$-equivariant diffeomorphisms replaces the group $\operatorname{Diff}(M, \omega)$ of $\omega$-invariant diffeomorphisms. The flux homomorphism integrating the infinitesimal flux homomorphism

$$
\text { flux : } \mathfrak{X}(M, \omega) \rightarrow H^{1}(M, V), \quad \text { flux }(\eta)=\left[i_{\eta} \omega\right]
$$

to $\operatorname{Diff}(M, \omega)$ can be extended to a flux 1-cocycle on $\operatorname{Diff}^{\text {eq }}(M, \omega)$, integrating the infinitesimal flux 1-cocycle

$$
\text { flux }^{\mathrm{eq}}:(\eta, \gamma) \in \mathfrak{X}^{\mathrm{eq}}(M, \omega) \mapsto\left[q^{*} i_{\eta} \omega-\gamma \cdot \theta\right] \in H^{1}(P, V) .
$$

The quantomorphisms are automorphisms of the principal bundle $P$, so the (gauge) abelian extension

$$
1 \rightarrow C^{\infty}(M, A) \rightarrow \operatorname{Diff}(P)^{A} \rightarrow \operatorname{Diff}(M)_{[P]} \rightarrow 1,
$$


contains the prequantization extension. In contrast to the quantomorphisms, the equi-quantomorphisms are not $A$-equivariant. Consequently, the gauge extension has to be enlarged. An extension of the group $\operatorname{Diff}(M)_{[P]}$ of diffeomorphisms preserving the isomorphism class of $P$ by the non-abelian group of almost $A$ invariant maps

$$
C_{A}^{\infty}(P, A)=\left\{f \in C^{\infty}(P, A): \forall a \in A, f^{-1}(f \circ \rho(a)) \text { constant on } P\right\}
$$

contains the prequantization abelian extension.

The plan of the paper is the following: Sect. 2 collects known results concerning central extensions; Sect. 3 introduces the flux 1-cocycle, a generalization of the symplectic flux homomorphism; Sect. 4 contains the construction of the prequantization abelian extension; in Sect. 5 we provide abelian Lie group extensions via prequantization; the case of an exact 2 -form $\omega$, when the extensions are given by group cocycles, is treated in Sect. 6, Sect. 7 is devoted to examples.

\section{Central extensions}

The prequantization extension associated to a prequantizable symplectic manifold $(M, \omega)$ is

$$
1 \rightarrow S^{1} \rightarrow \operatorname{Quant}(P, \theta)_{0} \rightarrow \operatorname{Ham}(M, \omega) \rightarrow 1 .
$$

for $P$ a principal circle bundle over $M$ with connection $\theta$ and curvature $\omega$ [7] [17]. In this section we present the prequantization extension in the more general case of a vector valued closed 2-form, following [11].

We consider a connected manifold $M$, possibly infinite dimensional, and we assume it is smoothly paracompact, in order to classify principal bundles over $M$ [3]. Let $\omega$ a closed 2-form on $M$ with values in the Mackey complete locally convex space $V$. The period group $\Gamma$ of $\omega$ is the image of the homomorphism $[\sigma] \in H_{2}(M, \mathbb{R}) \mapsto \int_{[\sigma]} \omega \in V$. We assume that the period group $\Gamma \subset V$ is discrete, i.e. $\omega$ is prequantizable. Let $\operatorname{Diff}(M, \omega)$ be the group of $\omega$-invariant diffeomorphisms and $\mathfrak{X}(M, \omega)$ the Lie algebra of $\omega$-invariant vector fields. The infinitesimal flux homomorphism

$$
\text { flux : } \mathfrak{X}(M, \omega) \rightarrow H^{1}(M, V), \quad \text { flux }(\eta)=\left[i_{\eta} \omega\right],
$$

can be integrated to the flux homomorphism [11]

$$
\begin{gathered}
\text { Flux : } \operatorname{Diff}(M, \omega)_{0} \rightarrow H^{1}(M, V) / H^{1}(M, \Gamma) \\
\operatorname{Flux}(\varphi)=\int_{0}^{1}\left[i_{\delta^{r} \varphi_{t}} \omega\right] d t \quad \bmod H^{1}(M, \Gamma)=\int_{0}^{1}\left[i_{\delta^{l} \varphi_{t}} \omega\right] d t \quad \bmod H^{1}(M, \Gamma),
\end{gathered}
$$

where $\varphi_{t}$ is any smooth curve in $\operatorname{Diff}(M, \omega)$ connecting the identity and $\varphi$, and $\delta^{l}, \delta^{r}$ denote the left, right logarithmic derivative (defined in the Appendix). The 
identity component $\operatorname{Diff}_{\mathrm{ex}}(M, \omega)$ of the kernel of the flux homomorphism Flux is called the group of exact $\omega$-invariant diffeomorphisms. As in [11, by the identity component $G_{0}$ of a group $G$ of diffeomorphisms of $M$ we understand the normal subgroup of those diffeomorphisms of $G$ connected to the identity by a curve $\varphi: I \rightarrow G$ such that the map $(t, x) \mapsto\left(\varphi(t) x, \varphi(t)^{-1} x\right)$ is smooth.

For a symplectic manifold $(M, \omega)$, the symplectic flux homomorphism $S_{\omega}$ involves a subgroup, $\Pi \subseteq H^{1}(M, \Gamma)$, called the flux subgroup, known to be discrete [12], so

$$
S_{\omega}: \operatorname{Diff}(M, \omega)_{0} \rightarrow H^{1}(M, \mathbb{R}) / \Pi, \quad S_{\omega}(\varphi)=\int_{0}^{1}\left[i_{\delta^{r} \varphi_{t}} \omega\right] d t \quad \bmod \Pi .
$$

In this case the group of hamiltonian diffeomorphisms $\operatorname{Ham}(M, \omega)$, defined as the group of those diffeomorphisms of $M$ which are endpoints of hamiltonian isotopies, is the kernel of the symplectic flux $S_{\omega}$ [1], and it coincides with $\operatorname{Diff}_{\mathrm{ex}}(M, \omega)$ [1]. By abuse of language we call $\operatorname{Diff}_{\mathrm{ex}}(M, \omega)$ the "hamiltonian" group even if $\omega$ is not symplectic.

Extensions of Lie algebras of vector fields. In this paragraph we present several Lie algebras of vector fields on a principal bundle: projectable vector fields, $A$-invariant vector fields, and infinitesimal quantomorphisms, as extensions of Lie algebras of vector fields on the base.

Let $A$ be the abelian Lie group $V / \Gamma$, where $\Gamma$ is the discrete period group of the closed vector valued 2 -form $\omega$. Since $M$ is smoothly paracompact, there exists a principal $A$-bundle $q: P \rightarrow M$ and a principal connection 1 -form $\theta \in \Omega^{1}(P, V)$ with curvature $\omega \in \Omega^{2}(M, V)$. The principal $A$-action is denoted by $\rho$, and the infinitesimal action by $\dot{\rho}: V \rightarrow \mathfrak{X}(P)$. In particular $d \theta=q^{*} \omega$ and $i_{\dot{\rho}(v)} \theta=v$ for all $v \in V$.

To every function $h \in C^{\infty}(P, V)$ one associates the vertical vector field $\dot{\rho}(h)$ on $P$ by $\dot{\rho}(h)(y)=\dot{\rho}(h(y))(y)$. We endow $C^{\infty}(P, V)$ with a Lie bracket such that the injective mapping $\dot{\rho}: C^{\infty}(P, V) \rightarrow \mathfrak{X}(P)$ becomes a Lie algebra homomorphism for the opposite Lie bracket on $\mathfrak{X}(P)$. This leads to

$$
\left[h_{1}, h_{2}\right]=L_{\dot{\rho}\left(h_{2}\right)} h_{1}-L_{\dot{\rho}\left(h_{1}\right)} h_{2},
$$

because $i_{\left[\dot{\rho}\left(h_{1}\right), \dot{\rho}\left(h_{2}\right)\right]} \theta=L_{\dot{\rho}\left(h_{1}\right)} h_{2}-L_{\dot{\rho}\left(h_{2}\right)} h_{1}$.

A vector field $\xi \in \mathfrak{X}(P)$ is called projectable if it is $q$-related to a vector field $\eta \in \mathfrak{X}(M)$, and we denote $\eta=q_{*} \xi$. Projectable vector fields can be characterized by $T q \circ \xi \circ \rho(a)=T q \circ \xi$ for all $a \in A$. Every vertical vector field is projectable and we get an exact sequence of Lie algebras

$$
0 \rightarrow C^{\infty}(P, V) \stackrel{\dot{\rho}}{\rightarrow} \mathfrak{X}_{\text {proj }}(P) \stackrel{q_{*}}{\rightarrow} \mathfrak{X}(M) \rightarrow 0 .
$$

The pull-back by $q$ maps $C^{\infty}(M, V)$ into an abelian Lie subalgebra of $C^{\infty}(P, V)$ because the bracket (4) on pull-back functions $q^{*} f$ for $f \in C^{\infty}(M, V)$ vanishes. 
The Lie algebra $\mathfrak{X}(P)^{A}$ of $A$-invariant vector fields on $P$ (infinitesimal automorphisms of $P$ ) consists of vector fields $\xi$ such that $\rho(a)^{*} \xi=\xi$ for all $a \in A$, or equivalently $L_{\dot{\rho}(v)} \xi=0$ for all $v \in V$. Restricting (5) to the Lie algebra $\mathfrak{X}(P)^{A}$, we obtain an abelian Lie algebra extension

$$
0 \rightarrow C^{\infty}(M, V) \stackrel{\dot{\rho}}{\rightarrow} \mathfrak{X}(P)^{A} \stackrel{q_{*}}{\rightarrow} \mathfrak{X}(M) \rightarrow 0 .
$$

Its cohomology class is given by the curvature form $\omega$ on $M$ viewed as a Lie algebra 2-cocycle on $\mathfrak{X}(M)$ with values in the $\mathfrak{X}(M)$-module $C^{\infty}(M, V)$.

An infinitesimal quantomorphism is an infinitesimal connection preserving automorphism of $P$. The Lie algebra of infinitesimal quantomorphisms can be expressed as

$$
\mathfrak{X}(P, \theta)^{A}=\left\{\xi \in \mathfrak{X}_{\text {proj }}(P): L_{\xi} \theta=0\right\}
$$

because if $q_{*} \xi=\eta$ and $L_{\xi} \theta=0$, then $[\xi, \dot{\rho}(v)]=0$ for all $v \in V$. Indeed, $[\xi, \dot{\rho}(v)]$ is a vertical vector field (q-related to $[\eta, 0]=0)$ and $i_{[\xi, \dot{\rho}(v)]} \theta=L_{\xi} v=0$, so $\xi \in \mathfrak{X}(P)^{A}$. Restricting ([6) further to $\mathfrak{X}(P, \theta)^{A}$, we get the prequantization central Lie algebra extension

$$
0 \rightarrow V \stackrel{\dot{\rho}}{\rightarrow} \mathfrak{X}(P, \theta)^{A} \stackrel{q_{*}}{\rightarrow} \mathfrak{X}_{\mathrm{ex}}(M, \omega) \rightarrow 0
$$

Indeed, let $\xi \in \mathfrak{X}(P, \theta)^{A}$. Both $\xi$ and $\theta \in \Omega^{1}(P, V)$ being $A$-invariant, the function $i_{\xi} \theta \in C^{\infty}(P, V)$ is $A$-invariant too, hence it descends to a function $q_{*} i_{\xi} \theta$ on $M$. Now $L_{\xi} \theta=0$ and $q_{*} \xi=\eta$ imply $i_{\eta} \omega=d\left(-q_{*} i_{\xi} \theta\right)$, so $\xi$ is $q$-related to the hamiltonian vector field $\eta$. On the other hand $\dot{\rho}(h) \in \mathfrak{X}(P, \theta)^{A}$ implies $0=L_{\dot{\rho}(h)} \theta=d h$, so all vertical infinitesimal quantomorphisms are of the form $\dot{\rho}(v), v \in V$. The cohomology class describing this extension is the class of the $V$-valued Lie algebra $2-$ cocycle on $\mathfrak{X}_{\mathrm{ex}}(M, \omega)$ given by $\left(\eta_{1}, \eta_{2}\right) \mapsto-\omega\left(\eta_{1}, \eta_{2}\right)\left(x_{0}\right), x_{0} \in M$.

Geometric prequantization. A function $f \in C^{\infty}(M, V)$ is a hamiltonian function for the vector field $\eta_{f}$ if $i_{\eta_{f}} \omega=d f$, so the hamiltonian functions on $M$ have to be constant along the leaves of Ker $\omega \subset T M$. They form the subspace of admissible functions $C_{\mathrm{adm}}^{\infty}(M, V)$. A hamiltonian vector field associated to such an admissible function $f$ can be determined only up to a section in $\Gamma(\operatorname{Ker} \omega)$.

Remark 2.1. The linear map

$$
\xi \in \mathfrak{X}(P, \theta)^{A} \mapsto-q_{*} i_{\xi} \theta \in C_{\mathrm{adm}}^{\infty}(M, V)
$$

is surjective with kernel $\Gamma(\operatorname{Ker} \omega)^{\text {hor }}$. By definition, the horizontal lift of a vector field $\eta \in \mathfrak{X}(M)$ is the unique vector field $\eta^{\text {hor }}, q$-related to $\eta$, satisfying $i_{\eta^{\text {hor }}} \theta=0$. Given an admissible function $f$, there exists a hamiltonian vector field $\eta_{f}$, and the vector field $\xi_{f}=\eta_{f}^{\text {hor }}-\dot{\rho}\left(q^{*} f\right)$ is an infinitesimal quantomorphism with $i_{\xi_{f}} \theta=$ $-q^{*} f$. 
In the symplectic case $\operatorname{Ker} \omega=0$, so we have $C_{\mathrm{adm}}^{\infty}(M)=C^{\infty}(M)$ and the hamiltonian vector field $\eta_{f}$ is uniquely determined by its hamiltonian function $f$. The linear map (8) is a bijection with inverse

$$
f \in C^{\infty}(M) \mapsto \xi_{f}:=\eta_{f}^{\text {hor }}-\left(q^{*} f\right) E \in \mathfrak{X}(P, \theta)^{A},
$$

$E=\dot{\rho}(1)$ denoting the infinitesimal generator of the circle action on $P$. This is the symplectic prequantization, in the construction due to Souriau [17].

Extensions of diffeomorphism groups. There is a bijection between $C^{\infty}(P, A)$ and the space $C_{\text {fiber }}^{\infty}(P, P)$ of fiber preserving smooth maps which associates to $f \in C^{\infty}(P, A)$ the map

$$
\rho(f): y \in P \mapsto \rho(f)(y)=\rho(y, f(y)) \in P .
$$

The composition on $C_{\text {fiber }}^{\infty}(P, P)$ determines a monoid structure on $C^{\infty}(P, A)$, namely

$$
\left(f_{1} \cdot f_{2}\right)(y)=f_{1}\left(\rho\left(y, f_{2}(y)\right)\right) f_{2}(y) .
$$

The image by $\rho$ of the group of invertible elements $C^{\infty}(P, A)_{\text {inver }}$ in $C^{\infty}(P, A)$ is the group of fiber preserving diffeomorphisms of $P$.

The group Diff proj $(P)$ of projectable diffeomorphisms is the group of diffeomorphisms of $P$ which map fibers to fibers, i.e. those $\psi \in \operatorname{Diff}(P)$ such that $q \circ \psi=\varphi \circ q$ for some $\varphi \in \operatorname{Diff}(M)$. Projectable diffeomorphisms of $P$ can be characterized by $q \circ \psi \circ \rho(a)=q \circ \psi$ for all $a \in A$. We write $\varphi=q_{*} \psi$ and the diffeomorphism $\varphi$ belongs to $\operatorname{Diff}(M)_{[P]}$, the group of diffeomorphisms preserving the isomorphism class $[P]$ of the principal bundle $P$. The exact sequence of groups

$$
1 \rightarrow C^{\infty}(P, A)_{\text {inver }} \stackrel{\rho}{\rightarrow} \operatorname{Diff}_{\text {proj }}(P) \stackrel{q_{*}}{\rightarrow} \operatorname{Diff}(M)_{[P]} \rightarrow 1
$$

is the global version of (5).

Let $\operatorname{Diff}(P)^{A}$ be the group of $A$-equivariant diffeomorphisms of $P$, i.e. the group of automorphisms of the principal bundle $P$. An abelian extension (the gauge extension) is obtained by restricting (11) to the subgroup $\operatorname{Diff}(P)^{A} \subset$ $\operatorname{Diff}_{\text {proj }}(P)$ :

$$
1 \rightarrow C^{\infty}(M, A) \stackrel{\rho}{\rightarrow} \operatorname{Diff}(P)^{A} \stackrel{q_{*}}{\rightarrow} \operatorname{Diff}(M)_{[P]} \rightarrow 1,
$$

with infinitesimal version the abelian Lie algebra extension (6).

A quantomorphisms is a connection preserving automorphism of $P$. The group of quantomorphisms can be expressed also as

$$
\operatorname{Diff}(P, \theta)^{A}=\left\{\psi \in \operatorname{Diff}_{\text {proj }}(P): \psi^{*} \theta=\theta\right\} .
$$

The quantomorphisms of $P$ descend to holonomy preserving diffeomorphisms on $M$. Denoting by $h(\ell) \in A$ the holonomy around a loop $\ell$ in $M$ for the principal connection $\theta$, let

$$
\operatorname{Hol}(M, \omega)=\left\{\varphi \in \operatorname{Diff}(M): \forall \ell \in C^{\infty}\left(S^{1}, M\right), h(\varphi \circ \ell)=h(\ell)\right\}
$$


be the group of holonomy preserving diffeomorphisms. It is a subgroup of the group $\operatorname{Diff}(M, \omega)$ of $\omega$-preserving diffeomorphisms.

A central extension can be obtained by a further restriction of (12) to the group of quantomorphisms [11]:

$$
1 \rightarrow A \stackrel{\rho}{\rightarrow} \operatorname{Diff}(P, \theta)^{A} \stackrel{q_{*}}{\rightarrow} \operatorname{Hol}(M, \omega) \rightarrow 1,
$$

An argument can be given using Proposition 8.4 in the Appendix: for $f \in$ $C^{\infty}(P, A), \rho(f)^{*} \theta=\theta$ if and only if $\delta^{l}(f)=0$, so $f$ is a constant $\in A$. Passing to connected components of the identity we get the prequantization central extension

$$
1 \rightarrow A \stackrel{\rho}{\rightarrow} \operatorname{Diff}(P, \theta)_{0}^{A} \stackrel{q_{*}}{\rightarrow} \operatorname{Diff}_{\mathrm{ex}}(M, \omega) \rightarrow 1,
$$

the analogue of (11) in the symplectic setting.

Geometric construction of central extensions. Let $G$ be a connected Lie group, $\lambda$ a smooth $G$-action on $M$ with infinitesimal action $\dot{\lambda}$, and $\omega \in \Omega^{2}(M, V)$ a $G$-invariant closed 2-form with discrete period group $\Gamma \subset V$. The $G$-action $\lambda$ is called a hamiltonian action if $i_{\dot{\lambda}(X)} \omega \in \Omega^{1}(M, V)$ is exact for all $X \in \mathfrak{g}$, so it gives a group homomorphism $\lambda: G \rightarrow \operatorname{Diff}_{\mathrm{ex}}(M, \omega)$.

In Theorem 3.4 from [1] is proven that, given a hamiltonian action $\lambda$ of a connected Lie group $G$ on $(M, \omega)$, there exists a central Lie group extension $\hat{G}$ of $G$ by $A=V / \Gamma$ and a smooth $\hat{G}$-action on $(P, \theta)$ by quantomorphisms, lifting the $G$-action. The central extension of $G$ is a pull-back of the prequantization central extension (14) and a corresponding Lie algebra cocycle on $\mathfrak{g}$ is $(X, Y) \mapsto$ $-\omega(\dot{\lambda}(X), \dot{\lambda}(Y))\left(x_{0}\right)$, where $x_{0} \in M$ is fixed. The manifold structure on $\hat{G}$ is obtained from the pull-back bundle of $P$ by an orbit map of $G$ on $M$.

\section{Flux 1-cocycle}

Let $M$ be a connected smoothly paracompact manifold, possibly infinite dimensional, $V$ a Mackey complete locally convex space, and $\omega \in \Omega^{2}(M, V)$ prequantizable, i.e. closed 2-form with discrete period group. In this section we introduce the group $\operatorname{Diffe}^{\text {eq }}(M, \omega)$ of $\omega$-equivariant diffeomorphisms as an isotropy subgroup of $\operatorname{Diff}(M) \times \mathrm{GL}(V)$, and we define a flux 1-cocycle, extending the flux homomorphism (2) on $\operatorname{Diff}(M, \omega)$. Properties of 1-cocycles can be found in the appendix.

The Lie algebra of $\omega$-equivariant vector fields on $M$,

$$
\mathfrak{X}^{\mathrm{eq}}(M, \omega):=\left\{(\eta, \gamma) \in \mathfrak{X}(M) \times \mathfrak{g l}(V): L_{\eta} \omega=\gamma \cdot \omega\right\},
$$

is the stabilizer of $\omega$ under the representation $(\eta, \gamma) \cdot \omega=-L_{\eta} \omega+\gamma \cdot \omega$ of the direct product Lie algebra $\mathfrak{X}(M) \times \mathfrak{g l}(V)$. We take the negative sign convention on Lie algebras of vector fields (so $\mathfrak{X}(M)$ is the Lie algebra of the group of 
diffeomorphisms of $M)$, so

$$
\left[\left(\eta_{1}, \gamma_{1}\right),\left(\eta_{2}, \gamma_{2}\right)\right]=\left(-\left[\eta_{1}, \eta_{2}\right], \gamma_{1} \gamma_{2}-\gamma_{2} \gamma_{1}\right) .
$$

The Lie algebra of $\omega$-invariant vector fields is a Lie subalgebra of $\mathfrak{X}^{\text {eq }}(M, \omega)$ for the inclusion

$$
\iota: \mathfrak{X}(M, \omega) \rightarrow \mathfrak{X}^{\mathrm{eq}}(M, \omega), \quad \iota(\eta)=(\eta, 0) .
$$

Remark 3.1. Let $V_{0}$ be the closure of the image of $\omega: T M \times_{M} T M \rightarrow V$. Given an $\omega$-equivariant vector field $(\eta, \gamma)$, the restriction of $\gamma$ to $V_{0}$ is uniquely determined by $\eta$. When $V_{0}=V$, then $\gamma$ is determined by $\eta$ and one can identify the Lie algebra $\mathfrak{X}^{\mathrm{eq}}(M, \omega)$ with its projection on the first factor: $\{\eta \in \mathfrak{X}(M): \exists \gamma \in$ $\mathfrak{g l}(V)$ s.t. $\left.L_{\eta} \omega=\gamma \cdot \omega\right\}$. When $V_{0} \neq V$, an $\omega$-invariant vector field $\eta$ can determine other $\omega$-equivariant vector fields beside $(\eta, 0)$, namely $(\eta, \gamma)$ with $\left.\gamma\right|_{V_{0}}=0$.

The period group $\Gamma$ is a subgroup of $V_{0}$ and, for any $(\eta, \gamma) \in \mathfrak{X}^{\mathrm{eq}}(M, \omega)$, the restriction of $\gamma$ to $\Gamma$ is trivial. Indeed, $\gamma\left(\int_{\sigma} \omega\right)=\int_{\sigma} L_{\eta} \omega=0$ for any 2-cycle $\sigma$ in $M$. In particular $\gamma=0$ if $V$ is generated by $\Gamma$, so $\iota(\mathfrak{X}(M, \omega))=\mathfrak{X}^{\text {eq }}(M, \omega)$ in this case. For a closed $\mathbb{R}$-valued 2 -form $\omega$, the Lie algebra of $\omega$-equivariant vector fields is strictly bigger than the Lie algebra of $\omega$-invariant vector fields if and only if $\Gamma=0$, i.e. $\omega$ is exact.

Infinitesimal flux cocycle. The projection on the second factor,

$$
(\eta, \gamma) \in \mathfrak{X}^{\mathrm{eq}}(M, \omega) \mapsto \gamma \in \mathfrak{g l}(V),
$$

is a Lie algebra homomorphism, so $V$ becomes a $\mathfrak{X}^{\text {eq }}(M, \omega)$-module in a natural way. The action of the image of $\iota$ is trivial.

Since the period group $\Gamma \subset V$ of $\omega$ is discrete, we consider again a principal $A=V / \Gamma$-bundle $q: P \rightarrow M$ with connection form $\theta \in \Omega^{1}(P, V)$ and curvature form $\omega \in \Omega^{2}(M, V)$.

Proposition 3.2. The linear map

$$
\text { flux }^{\mathrm{eq}}:(\eta, \gamma) \in \mathfrak{X}^{\mathrm{eq}}(M, \omega) \mapsto\left[q^{*} i_{\eta} \omega-\gamma \cdot \theta\right] \in H^{1}(P, V)
$$

is a Lie algebra 1-cocycle for the natural $\mathfrak{X}^{\mathrm{eq}}(M, \omega)$-module structure on $H^{1}(P, V)$ induced by the $\mathfrak{X}^{\mathrm{eq}}(M, \omega)$-action (16) on $V$.

Its cohomology class $\left[\mathrm{flux}{ }^{\mathrm{eq}}\right] \in H^{1}\left(\mathfrak{X}^{\mathrm{eq}}(M, \omega), H^{1}(P, V)\right)$ is independent of the choice of the connection $\theta$.

Proof. The 1 -form $q^{*} i_{\eta} \omega-\gamma \cdot \theta$ on $P$ is closed for any $\omega$-equivariant vector field $(\eta, \gamma)$ because $L_{\eta} \omega=\gamma \cdot \omega$ and $q^{*} \omega=d \theta$. For $\left(\eta_{1}, \gamma_{1}\right),\left(\eta_{2}, \gamma_{2}\right) \in \mathfrak{X}^{\mathrm{eq}}(M, \omega)$,

$$
\begin{aligned}
\operatorname{flux}^{\mathrm{eq}}\left(\left[\left(\eta_{1}, \gamma_{1}\right),\right.\right. & \left.\left.,\left(\eta_{2}, \gamma_{2}\right)\right]\right)=-\left[q^{*} i_{\left[\eta_{1}, \eta_{2}\right.} \omega+\left(\gamma_{1} \gamma_{2}-\gamma_{2} \gamma_{1}\right) \cdot \theta\right] \\
& =\left[d\left(q^{*} \omega\left(\eta_{1}, \eta_{2}\right)\right)+q^{*} i_{\eta_{2}} L_{\eta_{1}} \omega-q^{*} i_{\eta_{1}} L_{\eta_{2}} \omega-\gamma_{1} \gamma_{2} \cdot \theta+\gamma_{2} \gamma_{1} \cdot \theta\right] \\
& =\left[q^{*} i_{\eta_{2}}\left(\gamma_{1} \cdot \omega\right)-\gamma_{1} \gamma_{2} \cdot \theta\right]-\left[q^{*} i_{\eta_{1}}\left(\gamma_{2} \cdot \omega\right)-\gamma_{2} \gamma_{1} \cdot \theta\right] \\
& =\gamma_{1} \cdot \operatorname{flux}^{\mathrm{eq}}\left(\eta_{2}, \gamma_{2}\right)-\gamma_{2} \cdot \operatorname{flux}^{\mathrm{eq}}\left(\eta_{1}, \gamma_{1}\right),
\end{aligned}
$$


so the $1-$ cocycle condition for flux ${ }^{\text {eq }}$ is satisfied for the natural $\mathfrak{X}^{\mathrm{eq}}(M, \omega)$-action.

Two connection 1 -forms on $P$ differ by the pull-back $q^{*} \alpha$ of a closed $V$-valued 1-form $\alpha$ on $M$. Then the corresponding flux 1-cocycles differ by the linear map $(\eta, \gamma) \mapsto \gamma \cdot\left[q^{*} \alpha\right]$, which is a 1 -coboundary on the Lie algebra $\mathfrak{X}^{\mathrm{eq}}(M, \omega)$.

The 1-cocycle flux ${ }^{\mathrm{eq}}$ is called the infinitesimal flux 1-cocycle. Its kernel, denoted by $\mathfrak{X}_{\mathrm{ex}}^{\mathrm{eq}}(M, \omega)$, is a Lie subalgebra of $\mathfrak{X}^{\mathrm{eq}}(M, \omega)$ and is called the Lie algebra of equi-hamiltonian vector fields on $(M, \omega)$. We say that $h \in C^{\infty}(P, V)$ is an equi-hamiltonian function for the equi-hamiltonian vector field $(\eta, \gamma) \in \mathfrak{X}_{\mathrm{ex}}^{\mathrm{eq}}(M, \omega)$ if

$$
q^{*} i_{\eta} \omega-\gamma \cdot \theta=d h
$$

The equi-hamiltonian function doesn't determine uniquely the equi-hamiltonian vector field, unless $\operatorname{ker} \omega=0$.

Remark 3.3. The infinitesimal flux homomorphism (2) and the infinitesimal flux 1-cocycle (17) are related by flux ${ }^{\mathrm{eq}} \circ \iota=q^{*} \circ$ flux, hence the inclusion $\iota$ descends to an inclusion $\iota: \mathfrak{X}_{\mathrm{ex}}(M, \omega) \rightarrow \mathfrak{X}_{\mathrm{ex}}^{\mathrm{eq}}(M, \omega)$ of the ideal of exact $\omega$-invariant vector fields (hamiltonian vector fields when $\omega$ is symplectic) in the Lie algebra of equihamiltonian vector fields.

Admissible functions. Not every smooth $V$-valued function on $P$ can play the role of an equi-hamiltonian function. We denote by $C_{\mathrm{adm}}^{\infty}(P, V)$ the space of all possible equi-hamiltonian functions, also called admissible functions.

Proposition 3.4. If $h \in C_{\mathrm{adm}}^{\infty}(P, V)$ is an equi-hamiltonian function for the equihamiltonian vector field $(\eta, \gamma)$, then $L_{\dot{\rho}(v)} h=-\gamma(v)$ for all $v \in V$. There exists a group homomorphism $\bar{\gamma}: A \rightarrow V$ satisfying $\bar{\gamma} \circ \exp =\gamma$, with $\exp : V \rightarrow A$ the canonical projection, such that for all $a \in A$ holds $h-h \circ \rho(a)=\bar{\gamma}(a)$.

Proof. Since $h$ is an equi-hamiltonian function for the equi-hamiltonian vector field $(\eta, \gamma)$, we have that $d h=q^{*} i_{\eta} \omega-\gamma \cdot \theta$. Then from $L_{\dot{\rho}(v)} h=i_{\dot{\rho}(v)} d h=-\gamma(v)$ we get the first identity.

From $q \circ \rho(a)=q$ and $\rho(a)^{*} \theta=\theta$ it follows that $\rho(a)^{*} d h=d h$, so $h-h \circ \rho(a)$ is a constant function on the connected manifold $P$. This ensures the existence of a group homomorphism $\bar{\gamma}: A \rightarrow V$ satisfying the identity $h-h \circ \rho(a)=\bar{\gamma}(a)$ for all $a \in A$. From that we easily get that $\bar{\gamma} \circ \exp \in \mathfrak{g l}(V)$. To show that $\bar{\gamma} \circ \exp =\gamma$, we differentiate at $t=0$ the identity $h-h \circ \rho(\exp t v)=\bar{\gamma}(\exp t v)$ for $v \in V$, and we obtain that $L_{\dot{\rho}(v)} h=-T_{1} \bar{\gamma}(v)$. This gives $\gamma=T_{1} \bar{\gamma}=T_{0}(\bar{\gamma} \circ \exp )=\bar{\gamma} \circ \exp$.

The space of almost $A$-invariant functions is

$$
C_{A}^{\infty}(P, V)=\left\{h \in C^{\infty}(P, V): \forall a \in A, h-h \circ \rho(a)=\text { constant on } P\right\},
$$

so the proposition above says that $C_{\mathrm{adm}}^{\infty}(P, V) \subset C_{A}^{\infty}(P, V)$. It follows that for $h \in C_{A}^{\infty}(P, V)$ there exists a unique $\gamma_{h} \in \mathfrak{g l}(V)$ such that

$$
L_{\dot{\rho}(v)} h=-\gamma_{h}(v), \quad \forall v \in V .
$$


$\omega$-Equivariant diffeomorphisms. There is a natural $(\operatorname{Diff}(M) \times \mathrm{GL}(V))-$ action on the vector space $\Omega^{2}(M, V)$ of $V$-valued 2-forms on $M$ :

$$
(\varphi, u) \cdot \omega=u \cdot\left(\left(\varphi^{-1}\right)^{*} \omega\right)
$$

with infinitesimal action of the Lie algebra $\mathfrak{X}(M) \times \mathfrak{g l}(V)$ with Lie bracket (15) given by

$$
(\eta, \gamma) \cdot \omega=-L_{\eta} \omega+\gamma \cdot \omega
$$

The isotropy group of a closed 2-form $\omega \in \Omega^{2}(M, V)$ is the group of $\omega$-equivariant diffeomorphisms

$$
\operatorname{Diffeq}^{\mathrm{eq}}(M, \omega):=\left\{(\varphi, u) \in \operatorname{Diff}(M) \times \mathrm{GL}(V): \varphi^{*} \omega=u \cdot \omega\right\} .
$$

The isotropy Lie algebra coincides with the Lie algebra of $\omega$-equivariant vector fields $\mathfrak{X}^{\mathrm{eq}}(M, \omega)$.

The group of $\omega$-equivariant diffeomorphisms contains the group of $\omega$-invariant diffeomorphisms as a subgroup via the injective homomorphism

$$
i: \operatorname{Diff}(M, \omega) \rightarrow \operatorname{Diff}^{e q}(M, \omega), \quad i(\varphi)=\left(\varphi, 1_{V}\right) .
$$

The restriction of the second component $u \in \mathrm{GL}(V)$ of the $\omega$-equivariant diffeomorphism $(\varphi, u)$ to $V_{0} \subseteq V$ (defined in Remark 3.1) is determined by its first component $\varphi \in \operatorname{Diff}(M)$. When $V_{0}=V$, then one identifies $\operatorname{Diff}^{\text {eq }}(M, \omega)$ with its projection on the first factor, the group $\left\{\varphi \in \operatorname{Diff}(M): \exists u \in \mathrm{GL}(V)\right.$ s.t. $\left.\varphi^{*} \omega=u \cdot \omega\right\}$.

A curve $\varphi$ in $\operatorname{Diff}(M)$ is called a smooth curve if the corresponding map $(t, x) \mapsto$ $\left(\varphi(t)(x), \varphi(t)^{-1}(x)\right)$ in $M \times M$ is smooth. Similarly a curve $u$ in $\mathrm{GL}(V)$ is smooth if the map $(t, v) \mapsto\left(u(t)(v), u(t)^{-1}(v)\right)$ in $V \times V$ is smooth. Let $\operatorname{Diffe}^{\text {eq }}(M, \omega)_{0}$ be the normal subgroup of those elements in $\operatorname{Diff}^{\text {eq }}(M, \omega)$ which can be connected to the identity by a smooth curve in $\operatorname{Diff}^{\text {eq }}(M, \omega) \subset \operatorname{Diff}(M) \times \mathrm{GL}(V)$.

Remark 3.5. The second projection $(\varphi, u) \in \operatorname{Diffeq}^{e}(M, \omega) \mapsto u \in \operatorname{GL}(V)$ is a group homomorphism, so $V$ becomes a natural $\operatorname{Diff}^{\text {eq }}(M, \omega)$-module. The abelian group $A=V / \Gamma$ is a $\operatorname{Diffeq}^{\text {eq }}(M, \omega)_{0}$-module too. Indeed, for any 2 -cycle $\sigma$ in $M$ and for any $(\varphi, u) \in \operatorname{Diff}^{\mathrm{eq}}(M, \omega)_{0}$, the 2-cycles $\sigma$ and $\varphi(\sigma)$ are homologous, so $u\left(\int_{\sigma} \omega\right)=\int_{\sigma} \varphi^{*} \omega=\int_{\sigma} \omega$ and $u$ fixes the elements of the period group $\Gamma$. In particular $u$ descends to a group automorphism $\bar{u}$ of $A$, and the $\operatorname{Diffeq}^{\mathrm{eq}}(M)$-action on $V$ descends to an action on the abelian group $A$. If $V$ is generated by $\Gamma$, then $u=1_{V}$ and $\varphi$ is $\omega$-invariant for all $\omega$-equivariant diffeomorphisms $(\varphi, u)$.

Proposition 3.6. The following equivalences hold for smooth paths $\varphi_{t}$ in $\operatorname{Diff}(M)$ and $u_{t}$ in $\operatorname{GL}(V)$ starting at the identity: $\left(\varphi_{t}, u_{t}\right) \in \operatorname{Diff}^{\mathrm{eq}}(M, \omega) \Leftrightarrow\left(\delta^{l} \varphi_{t}, \delta^{l} u_{t}\right) \in$ $\mathfrak{X}^{\mathrm{eq}}(M, \omega) \Leftrightarrow\left(\delta^{r} \varphi_{t}, \delta^{r} u_{t}\right) \in \mathfrak{X}^{\mathrm{eq}}(M, \omega)$.

This follows from Remark 8.2 in the Appendix. In particular if the flow of an $\omega$-equivariant vector field $(\eta, \gamma)$ exists, then it consists of $\omega$-equivariant diffeomorphisms. 
Lemma 3.7. For any loop $\ell$ in $P$ and any smooth path of $\omega$-equivariant diffeomorphisms $\left(\varphi_{t}, u_{t}\right)$ starting at the identity, we define the 2-chain $\sigma$ swept out by the loop $q \circ \ell$ in $M$ under the isotopy $\varphi_{t}$, i.e. $\sigma(t, s)=\varphi_{t}(q(\ell(s))), t, s \in[0,1]$. Then

$$
\int_{\ell} \int_{0}^{1} u_{t} \cdot\left(q^{*} i_{\delta^{l} \varphi_{t}} \omega-\delta^{l} u_{t} \cdot \theta\right) d t=\int_{\sigma} \omega-u \cdot \int_{\ell} \theta+\int_{\ell} \theta
$$

Proof. Using $\varphi_{t}^{*} \omega=u_{t} \cdot \omega$, we compute

$$
\begin{aligned}
\int_{0}^{1}\left(u_{t}\right. & \left.\cdot \int_{\ell} q^{*} i_{\delta^{l} \varphi_{t}} \omega\right) d t=\int_{0}^{1} u_{t} \cdot\left(\int_{0}^{1}\left(q^{*} i_{\delta^{l} \varphi_{t}} \omega\right)(\dot{\ell}(s)) d s\right) d t \\
& =\int_{0}^{1} \int_{0}^{1} u_{t} \cdot \omega\left(\delta^{l} \varphi_{t}(q(\ell(s))), T q \cdot \dot{\ell}(s)\right) d s d t \\
& =\int_{0}^{1} \int_{0}^{1} \omega\left(\dot{\varphi}_{t}(q(\ell(s))), T \varphi_{t} \cdot T q \cdot \dot{\ell}(s)\right) d s d t=\int_{\sigma} \omega
\end{aligned}
$$

and the result follows.

Flux cocycle. The quotient space $H^{1}(P, V) / H^{1}(P, \Gamma)$ receives a natural Diffeq $(M, \omega)_{0^{-}}$ module structure from the action on the range $V$. Indeed, as we have seen in Remark 3.5, $\operatorname{Diff}^{\mathrm{eq}}(M, \omega)_{0}$ acts trivially on $\Gamma \subset V$, hence it acts trivially on $H^{1}(P, \Gamma) \subset H^{1}(P, V)$.

The map

$$
\begin{aligned}
& \operatorname{Flux}^{\mathrm{eq}}: \operatorname{Diff}^{\mathrm{eq}}(M, \omega)_{0} \rightarrow H^{1}(P, V) / H^{1}(P, \Gamma) \\
& \operatorname{Flux}^{\mathrm{eq}}(\varphi, u)=\int_{0}^{1} u_{t} \cdot \mathrm{flux}^{\mathrm{eq}}\left(\delta^{l} \varphi_{t}, \delta^{l} u_{t}\right) d t \quad \bmod H^{1}(P, \Gamma) \\
& =\left[\int_{0}^{1} u_{t} \cdot\left(q^{*} i_{\delta^{l} \varphi_{t}} \omega-\delta^{l} u_{t} \cdot \theta\right) d t\right] \bmod H^{1}(P, \Gamma),
\end{aligned}
$$

for any piecewise smooth path of $\omega$-equivariant diffeomorphisms $\left(\varphi_{t}, u_{t}\right)$ from the identity to $(\varphi, u)$, is a well defined group 1-cocycle, called the flux 1-cocycle associated to the closed vector valued form $\omega$ with discrete period group $\Gamma$.

The map Flux ${ }^{\text {eq }}$ is well defined because Lemma 3.7 implies that for a loop $\left(\varphi_{t}, u_{t}\right)$ of $\omega$-equivariant diffeomorphisms based at the identity, the integral over a loop $\ell$ in $P$ of the 1 -form $\int_{0}^{1} u_{t} \cdot\left(q^{*} i_{\delta^{l} \varphi_{t}} \omega-\delta^{l} u_{t} \cdot \theta\right) d t$ is the integral of $\omega$ over a 2 -cycle $\sigma$, hence it belongs to the group $\Gamma$ of periods of $\omega$. The 1 -cocycle condition for Flux ${ }^{\text {eq }}$ is verified as in Proposition 8.6 from the Appendix.

Remark 3.8. The group Diff ex $(M, \omega)=\left(\operatorname{Ker~Flux}_{\mathrm{ex}}^{\mathrm{eq}}\right)_{0}$ is called the group of equihamiltonian diffeomorphisms. The flux 1-cocycle Flux ${ }^{\mathrm{eq}}$ and the flux homomorphism Flux are related by Flux ${ }^{\mathrm{eq}} \circ i=q^{*} \circ$ Flux, hence $i$ descends to an injective homomorphism $\left.\operatorname{Diff}_{\mathrm{ex}}(M, \omega)\right) \rightarrow \operatorname{Diffex}_{\mathrm{ex}}^{\mathrm{eq}}(M, \omega)$.

The next proposition follows from Remark 8.9 in the Appendix. 
Proposition 3.9. For any piecewise smooth path $\left(\varphi_{t}, u_{t}\right)$ of $\omega$-equivariant diffeomorphisms, we have $\left(\varphi_{t}, u_{t}\right) \in \operatorname{Diff}_{\mathrm{ex}}^{\mathrm{eq}}(M, \omega) \Leftrightarrow\left(\delta^{l} \varphi_{t}, \delta^{l} u_{t}\right) \in \mathfrak{X}_{\mathrm{ex}}^{\mathrm{eq}}(M, \omega) \Leftrightarrow\left(\delta^{r} \varphi_{t}, \delta^{r} u_{t}\right) \in$ $\mathfrak{X}_{\mathrm{ex}}^{\mathrm{eq}}(M, \omega)$.

Remark 3.10. In the special case when $\omega=d \alpha$ for an $\alpha \in \Omega^{1}(M, V)$ (in particular the period group $\Gamma$ is trivial), the flux homomorphism is given by $\operatorname{Flux}(\varphi)=$ $\left[\varphi^{*} \alpha-\alpha\right] \in H^{1}(M, V)$. To compute the flux 1-cocycle Flux ${ }^{\mathrm{eq}}$ in this case, let $P=M \times V \stackrel{q}{\rightarrow} M$ be the trivial $V$-bundle with principal connection 1-form $\theta=q^{*} \alpha+\theta_{V}$ and curvature $\omega$, where $\theta_{V}=\delta^{l}\left(1_{V}\right) \in \Omega^{1}(V, V)$ stands for the Maurer-Cartan form on $V$. We get

$$
\text { Flux }^{\mathrm{eq}}: \operatorname{Diffeq}^{\mathrm{eq}}(M, \omega) \rightarrow H^{1}(P, V), \quad \operatorname{Flux}^{\mathrm{eq}}(\varphi, u)=q^{*}\left[\varphi^{*} \alpha-u \cdot \alpha\right] .
$$

Indeed, let $\left(\varphi_{t}, u_{t}\right)$ be a path of $\omega$-equivariant diffeomorphisms joining the identity and $(\varphi, u)$. From $\frac{d}{d t}\left[\varphi_{t}^{*} \alpha\right]=\left[u_{t} \cdot i_{\delta^{l}} \varphi_{t} \omega\right]$ we obtain $\frac{d}{d t}\left[q^{*} \varphi_{t}^{*} \alpha-u_{t} \cdot \theta\right]=u_{t} \cdot\left[q^{*} i_{\delta^{l}} \varphi_{t} \omega-\right.$ $\left.\delta^{l} u_{t} \cdot \theta\right]$. Integrating this cohomology class from 0 to 1 gives the expression of the flux cocycle $\operatorname{Flux}^{\mathrm{eq}}(\varphi, u)=q^{*}\left[\varphi^{*} \alpha-u \cdot \alpha\right]-\left[u \cdot \theta_{V}-\theta_{V}\right]=q^{*}\left[\varphi^{*} \alpha-u \cdot \alpha\right]$, because $\theta_{V}$ is an exact 1 -form on $P$.

\section{Prequantization abelian extension}

Appropriate prequantization procedures have been developed for symplectic, presymplectic, Poisson

and Dirac manifolds [18]. In this section we suggest a prequantization procedure for a closed vector valued 2 -form in the equivariant setting.

Infinitesimal equi-quantomorphisms. The space $C_{A}^{\infty}(P, V)$ of almost $A$ invariant functions defined in (19) endowed with the Lie bracket

$$
\left[h_{1}, h_{2}\right]=\gamma_{h_{2}} \circ h_{1}-\gamma_{h_{1}} \circ h_{2},
$$

is a Lie subalgebra of $C^{\infty}(P, V)$ with Lie bracket (4), since from (20) follows that $L_{\dot{\rho}\left(h_{1}\right)} h_{2}=-\gamma_{h_{2}} \circ h_{1}$ for $h_{1}, h_{2} \in C_{A}^{\infty}(P, V)$.

$$
\mathfrak{X}^{\mathrm{eq}}(P)^{A}=\left\{\xi \in \mathfrak{X}(P): \exists \gamma \in \mathfrak{g l}(V) \text { s.t. } \forall v \in V, L_{\dot{\rho}(v)} \xi=\dot{\rho}(\gamma(v))\right\}
$$

is the Lie algebra of almost A-invariant vector fields. It can be characterized as

$$
\mathfrak{X}^{\mathrm{eq}}(P)^{A}=\left\{\xi \in \mathfrak{X}(P): \exists \bar{\gamma}: A \rightarrow V \text { s.t. } \forall a \in A, \rho(a)^{*} \xi-\xi=\dot{\rho}(\bar{\gamma}(a))\right\},
$$

because of the identity

$$
\frac{d}{d t}\left(\rho(\exp t v)^{*} \xi-\xi-\dot{\rho}(\bar{\gamma}(\exp t v))\right)=\rho(\exp t v)^{*}\left(L_{\dot{\rho}(v)} \xi-\dot{\rho}(\gamma(v))\right),
$$

where $\bar{\gamma}$ is a group homomorphism with $\bar{\gamma} \circ \exp =\gamma$. In particular every almost $A$-invariant vector field is projectable. 
Proposition 4.1. When restricting the extension (5) to the Lie algebra $\mathfrak{X}^{\mathrm{eq}}(P)^{A}$ of almost $A$-invariant vector fields, one obtains a new Lie algebra extension

$$
0 \rightarrow C_{A}^{\infty}(P, V) \stackrel{\dot{\rho}}{\rightarrow} \mathfrak{X}^{\mathrm{eq}}(P)^{A} \stackrel{q_{*}}{\rightarrow} \mathfrak{X}(M) \rightarrow 0,
$$

with $C_{A}^{\infty}(P, V)$ the space of almost $A$-invariant functions defined in (19).

Proof. For an arbitrary $h \in C^{\infty}(P, V)$, the necessary and sufficient condition for the vertical vector field $\dot{\rho}(h)$ to be almost $A$-invariant is $L_{\dot{\rho}(v)} \dot{\rho}(h)=\dot{\rho}(\gamma(v))$. But we know from (4) that $L_{\dot{\rho}(v)} \dot{\rho}(h)=-\dot{\rho}\left(L_{\dot{\rho}(v)} h\right)$, so the condition above becomes $L_{\dot{\rho}(v)} h=-\gamma(v)$, which means $h \in C_{A}^{\infty}(P, V)$.

To pass to an abelian extension by $V$, we have to consider the Lie algebra

$$
\mathfrak{X}^{\mathrm{eq}}(P, \theta)^{A}=\left\{\xi \in \mathfrak{X}_{\text {proj }}(P): \exists \gamma_{\xi} \in \mathfrak{g l}(V) \text { s.t. } L_{\xi} \theta=\gamma_{\xi} \cdot \theta\right\}
$$

of infinitesimal equi-quantomorphisms. The linear map $\gamma_{\xi} \in \mathfrak{g l}(V)$ is determined by $\xi$ because $\gamma_{\xi}(v)=\gamma_{\xi}\left(i_{\dot{\rho}(v)} \theta\right)=i_{\dot{\rho}(v)} L_{\xi} \theta$. The Lie algebra $\mathfrak{X}^{\mathrm{eq}}(P, \theta)^{A}$ contains as a Lie subalgebra the Lie algebra $\mathfrak{X}(P, \theta)^{A}$ of infinitesimal quantomorphisms.

An equivariant version of Remark 2.1 holds.

Proposition 4.2. The linear map

$$
\xi \in \mathfrak{X}^{\mathrm{eq}}(P, \theta)^{A} \mapsto-i_{\xi} \theta \in C_{\mathrm{adm}}^{\infty}(P, V) .
$$

is surjective with kernel $\Gamma(\operatorname{Ker} \omega)^{\text {hor }}$.

Proof. Given $h \in C_{\mathrm{adm}}^{\infty}(P, V)$, let $(\eta, \gamma)$ be an equi-hamiltonian vector field with equi-hamiltonian function $h$ and let $\xi:=\eta^{\text {hor }}-\dot{\rho}(h)$. Then $\xi \in \mathfrak{X}^{\mathrm{eq}}(P, \theta)^{A}$ because $L_{\xi} \theta=L_{\eta^{\text {hor }}} \theta-L_{\dot{\rho}(h)} \theta=q^{*} i_{\eta} \omega-d h=\gamma \cdot \theta$. The linear correspondence (26) is surjective since $-i_{\xi} \theta=h$.

Let $\xi \in \mathfrak{X}^{\mathrm{eq}}(P, \theta)^{A}$ be an element in the kernel of (26). Then $\xi$ is a horizontal lift: there exists $\eta \in \mathfrak{X}(M)$ such that $\xi=\eta^{\text {hor }}$. But $\xi$ is an infinitesimal quantomorphism, so $\gamma_{\xi} \cdot \theta=L_{\eta^{\text {hor }}} \theta=i_{\eta_{\text {hor }}} d \theta=q^{*} i_{\eta} \omega$. Evaluating on vertical vectors we get $\gamma_{\xi}=0$, so $i_{\eta} \omega=0$, which means $\eta \in \Gamma(\operatorname{Ker} \omega)$.

The next proposition shows the inclusion $\mathfrak{X}^{\mathrm{eq}}(P, \theta)^{A} \subset \mathfrak{X}^{\mathrm{eq}}(P)^{A}$.

Proposition 4.3. Any infinitesimal equi-quantomorphism with $L_{\xi} \theta=\gamma_{\xi} \cdot \theta$ satisfies $L_{\dot{\rho}(v)} \xi=\dot{\rho}\left(\gamma_{\xi}(v)\right)$ for all $v \in V$.

Proof. The infinitesimal equi-quantomorphism $\xi$ is projectable and $\dot{\rho}(v)$ is vertical, so $L_{\dot{\rho}(v)} \xi=[\dot{\rho}(v), \xi]$ is also vertical. A short computation using $L_{\xi} \theta=\gamma_{\xi} \cdot \theta$ gives $i_{[\dot{\rho}(v), \xi]} \theta=\gamma_{\xi}(v)$. These two facts imply $L_{\dot{\rho}(v)} \xi=\dot{\rho}\left(\gamma_{\xi}(v)\right)$. 
The infinitesimal equi-quantomorphism $\xi$ determines an $\omega$-equivariant vector field $\left(q_{*} \xi, \gamma_{\xi}\right)$ on $M$. Moreover $\left(q_{*} \xi, \gamma_{\xi}\right)$ is an equi-hamiltonian vector field for the equi-hamiltonian function $h=-i_{\xi} \theta \in C_{\mathrm{adm}}^{\infty}(P, V)$, because

$$
d h=-d i_{\xi} \theta=i_{\xi} d \theta-L_{\xi} \theta=q^{*} i_{q_{*} \xi} \omega-\gamma_{\xi} \cdot \theta .
$$

Theorem 4.4. The Lie algebra of infinitesimal equi-quantomorphisms is an abelian extension of the Lie algebra $\mathfrak{X}_{\mathrm{ex}}^{\mathrm{eq}}(M, \omega)$ of equi-hamiltonian vector fields by the natural $\mathfrak{X}_{\mathrm{ex}}^{\mathrm{eq}}(M, \omega)$-module $V$. An abelian Lie algebra 2 -cocycle on $\mathfrak{X}_{\mathrm{ex}}^{\mathrm{eq}}(M, \omega)$ defining this abelian extension is $\left(\left(\eta_{1}, \gamma_{1}\right),\left(\eta_{2}, \gamma_{2}\right)\right) \mapsto-\omega\left(\eta_{1}, \eta_{2}\right)\left(x_{0}\right)$, for any fixed element $x_{0} \in M$.

Proof. We show that the following sequence of Lie algebras is exact:

$$
0 \rightarrow V \stackrel{\dot{\rho}}{\rightarrow} \mathfrak{X}^{\mathrm{eq}}(P, \theta)^{A} \stackrel{p}{\rightarrow} \mathfrak{X}_{\mathrm{ex}}^{\mathrm{eq}}(M, \omega) \rightarrow 0,
$$

where $p(\xi)=\left(q_{*} \xi, \gamma_{\xi}\right)$. The injectivity of $\dot{\rho}$ is clear. For the surjectivity of $p$ we consider an equi-hamiltonian vector field $(\eta, \gamma)$ with equi-hamiltonian function $h \in C^{\infty}(P, V)$. Then the infinitesimal equi-quantomorphism $\xi=\eta^{\text {hor }}-\dot{\rho}(h)$ (from the proof of Proposition 4.2) projects to $(\eta, \gamma)$.

The inclusion $\dot{\rho}(V) \subseteq \operatorname{Ker} p$ follows from $p \circ \dot{\rho}=0$. For the reversed inclusion let $\xi \in \operatorname{Ker} p \subset \mathfrak{X}^{\mathrm{eq}}(P, \theta)^{A}$. Then $L_{\xi} \theta=0$ and $\xi=\dot{\rho}(h)$ for some $h \in C^{\infty}(P, V)$. From $L_{\dot{\rho}(h)} \theta=d h$ and from the connectedness of $P$ follows that $\xi \in \dot{\rho}(V)$. The induced action of $\mathfrak{X}_{\mathrm{ex}}^{\mathrm{eq}}(M, \omega)$ on $V$ is the natural one because from Proposition 4.3 we get $[\dot{\rho}(v), \xi]=\dot{\rho}\left(\gamma_{\xi}(v)\right)$ for all $v \in V$ and $\xi \in \mathfrak{X}^{\mathrm{eq}}(P, \theta)^{A}$.

We determine the 2 -cocycle defined with the linear section $s$ of (27) given by $s(\eta, \gamma)=\eta^{\text {hor }}-\dot{\rho}(h)$, where $h$ is the unique equi-hamiltonian function of the equi-hamiltonian vector field $(\eta, \gamma)$ vanishing at a fixed point $y_{0} \in q^{-1}\left(x_{0}\right)$. First we observe that given the equi-hamiltonian vector fields $\left(\eta_{1}, \gamma_{1}\right)$ and $\left(\eta_{2}, \gamma_{2}\right)$ with equi-hamiltonian functions $h_{1}$ and $h_{2}$ vanishing at $y_{0}$, the equi-hamiltonian function vanishing at $y_{0}$ for the bracket $\left(-\left[\eta_{1}, \eta_{2}\right], \gamma_{1} \gamma_{2}-\gamma_{2} \gamma_{1}\right)$ is $\gamma_{1} \circ h_{2}-\gamma_{2} \circ$ $h_{1}+q^{*} \omega\left(\eta_{1}, \eta_{2}\right)-\omega\left(\eta_{1}, \eta_{2}\right)\left(x_{0}\right)$. Indeed,

$$
\begin{aligned}
d\left(\gamma_{1} \circ h_{2}-\gamma_{2} \circ h_{1}\right. & \left.+q^{*} \omega\left(\eta_{1}, \eta_{2}\right)\right) \\
& \stackrel{[11]}{=} \gamma_{1} \cdot\left(q^{*} i_{\eta_{2}} \omega-\gamma_{2} \cdot \theta\right)-\gamma_{2} \cdot\left(q^{*} i_{\eta_{1}} \omega-\gamma_{1} \cdot \theta\right)+q^{*} d i_{\eta_{2}} i_{\eta_{1}} \omega \\
& =q^{*}\left(i_{\eta_{2}} L_{\eta_{1}} \omega-i_{\eta_{1}} L_{\eta_{2}} \omega+d i_{\eta_{2}} i_{\eta_{1}} \omega\right)-\left(\gamma_{1} \circ \gamma_{2}-\gamma_{2} \circ \gamma_{1}\right) \cdot \theta \\
& =-q^{*} i_{\left[\eta_{1}, \eta_{2}\right]} \omega-\left(\gamma_{1} \circ \gamma_{2}-\gamma_{2} \circ \gamma_{1}\right) \cdot \theta .
\end{aligned}
$$

Since $\omega$ is the curvature of the principal bundle $P$, the identity $\left[\eta_{1}^{\text {hor }}, \eta_{2}^{\text {hor }}\right]-$ $\left[\eta_{1}, \eta_{2}\right]^{\text {hor }}=-\dot{\rho}\left(q^{*} \omega\left(\eta_{1}, \eta_{2}\right)\right)$ holds. The equi-hamiltonian functions $h_{1}$ and $h_{2}$ belong to $C_{A}^{\infty}(P, V)$, so by (22) we have $\left[\dot{\rho}\left(h_{1}\right), \dot{\rho}\left(h_{2}\right)\right]=\dot{\rho}\left(\gamma_{1} \circ h_{2}-\gamma_{2} \circ h_{1}\right)$. Using also the fact that

$$
\left[\dot{\rho}\left(h_{1}\right), \eta_{2}^{\text {hor }}\right]=-\dot{\rho}\left(L_{\eta_{2}^{\text {hor }}} h_{1}\right) \stackrel{\sqrt{187}=}{=}-\dot{\rho}\left(q^{*} \omega\left(\eta_{1}, \eta_{2}\right)\right),
$$


we compute

$$
\begin{aligned}
{\left[s\left(\eta_{1}, \gamma_{1}\right), s\left(\eta_{2}, \gamma_{2}\right)\right] } & -s\left(\left[\left(\eta_{1}, \gamma_{1}\right),\left(\eta_{2}, \gamma_{2}\right)\right]\right)=-\left[\eta_{1}^{\text {hor }}-\dot{\rho}\left(h_{1}\right), \eta_{2}^{\text {hor }}-\dot{\rho}\left(h_{2}\right)\right] \\
& +\left[\eta_{1}, \eta_{2}\right]^{\text {hor }}-\dot{\rho}\left(\gamma_{2} \circ h_{1}-\gamma_{1} \circ h_{2}-q^{*} \omega\left(\eta_{1}, \eta_{2}\right)+\omega\left(\eta_{1}, \eta_{2}\right)\left(x_{0}\right)\right) \\
& =\left[\dot{\rho}\left(h_{1}\right), \eta_{2}^{\text {hor }}\right]-\left[\dot{\rho}\left(h_{2}\right), \eta_{1}^{\text {hor }}\right]-\left[\dot{\rho}\left(h_{1}\right), \dot{\rho}\left(h_{2}\right)\right] \\
& -\dot{\rho}\left(\gamma_{2} \circ h_{1}\right)+\dot{\rho}\left(\gamma_{1} \circ h_{2}\right)+2 \dot{\rho}\left(q^{*} \omega\left(\eta_{1}, \eta_{2}\right)\right)-\dot{\rho}\left(\omega\left(\eta_{1}, \eta_{2}\right)\left(x_{0}\right)\right) \\
& =-\dot{\rho}\left(\omega\left(\eta_{1}, \eta_{2}\right)\left(x_{0}\right)\right),
\end{aligned}
$$

thus obtaining a Lie algebra 2-cocycle for the abelian extension (27).

Remark 4.5. Under the assumption that the closure $V_{0}$ of the image of $\omega: T M \times_{M}$ $T M \rightarrow V$ is the whole $V$, the Lie algebra of equi-hamiltonian vector fields can be identified with its projection on the first factor:

$$
\mathfrak{X}_{\mathrm{ex}}^{\mathrm{eq}}(M, \omega)=\left\{\eta \in \mathfrak{X}(M): \exists \gamma \in \mathfrak{g l}(V) \text { s.t. } q^{*} i_{\eta} \omega-\gamma \cdot \theta \text { exact }\right\} .
$$

In this case the abelian extension (27) can be seen as a restriction of (25).

Prequantization abelian extension. We saw in Sect. 2 that the gauge extension (12) (of $A$-equivariant diffeomorphisms of $P$ ) is an abelian group extension which contains the prequantization central extension. In this paragraph we construct the prequantization abelian extension together with a non-abelian extension (of almost $A$-equivariant diffeomorphisms of $P$ ) containing it.

The group of almost $A$-equivariant diffeomorphisms of $P$ is

$$
\operatorname{Diff}_{A}(P)=\left\{\psi \in \operatorname{Diff}(P): \exists \bar{u}_{\psi} \in \operatorname{Aut}(A) \text { s.t. } \psi \circ \rho(a)=\rho\left(\bar{u}_{\psi}(a)\right) \circ \psi, \forall a \in A\right\},
$$

where $\operatorname{Aut}(A)$ denotes the group of automorphisms of the abelian group $A$. One can describe $\operatorname{Diff}_{A}(P)$ as the group of those diffeomorphisms $\psi$ of $P$ such that the vertical vector fields $\dot{\rho}(v)$ and $\dot{\rho}\left(u_{\psi}(v)\right)$ are $\psi$-related. It is a subgroup of the group $\operatorname{Diff}_{\text {proj }}(P)$ of projectable diffeomorphisms and it contains the group $\operatorname{Diff}(P)^{A}$ of equivariant diffeomorphisms of $P$ as a subgroup.

The fiber preserving diffeomorphism $\rho(f)$ for $f \in C^{\infty}(P, A)$ is almost $A$-equivariant if and only if $\rho(f) \circ \rho(a)=\rho\left(\bar{u}_{\rho(f)}(a)\right) \circ \rho(f)$, which can be written as $f(y)^{-1} f(\rho(y, a))=a^{-1} \bar{u}_{\rho(f)}(a)$ for all $y \in P$. We define the set of almost $A-$ invariant maps

$$
C_{A}^{\infty}(P, A)=\left\{f \in C^{\infty}(P, A): \forall a \in A, f^{-1}(f \circ \rho(a)) \text { constant on } P\right\} .
$$

For an almost $A$-invariant function $h \in C_{A}^{\infty}(P, V)$, exp $\circ h$ is an almost $A$-invariant map with $\bar{u}_{f}=\exp \circ \bar{\gamma}_{h}$. The existence of a unique $\bar{u}_{f} \in \operatorname{Aut}(A)$ such that $f(y)^{-1} f(\rho(y, a))=a^{-1} \bar{u}_{f}(a)$ for all $a \in A$ and $y \in P$ follows easily. We observe that $\rho(f) \in \operatorname{Diff}_{A}(P)$ if and only if $f$ is an almost $A$-invariant map with $\bar{u}_{f}=\bar{u}_{\rho(f)}$. 
With the group multiplication

$$
\left(f_{1} \cdot f_{2}\right)(y)=f_{1}(y) \bar{u}_{f_{1}}\left(f_{2}(y)\right),
$$

$C_{A}^{\infty}(P, A)$ becomes a subgroup of $C^{\infty}(P, A)_{\text {inver }}$ with multiplication (10). The abelian group $C^{\infty}(M, A)$, identified with the group of $A$-invariant maps $f: P \rightarrow$ $A$, is a subgroup of $C_{A}^{\infty}(P, A)$ (in this case $\bar{u}_{f}=1_{A}$ ).

Restricting (11) to almost $A$-equivariant diffeomorphisms, we obtain a nonabelian group extension with infinitesimal version (25)):

$$
1 \rightarrow C_{A}^{\infty}(P, A) \stackrel{\rho}{\rightarrow} \operatorname{Diff}_{A}(P) \stackrel{q_{*}}{\rightarrow} \operatorname{Diff}(M)_{[P]} \rightarrow 1 .
$$

This is an enlarged version of the gauge extension (12).

We define the group of equi-quantomorphisms as

$$
\operatorname{Diff}^{\text {eq }}(P, \theta)^{A}=\left\{\psi \in \operatorname{Diff}_{\text {proj }}(P): \exists u_{\psi} \in \mathrm{GL}(V) \text { s.t. } \psi^{*} \theta=u_{\psi} \cdot \theta\right\} .
$$

It contains the group $\operatorname{Diff}(P, \theta)^{A}$ of quantomorphisms as a subgroup. The linear isomorphism $u_{\psi}$ is uniquely determined by $\psi \in \operatorname{Diffeq}^{\text {eq }}(P, \theta)^{A}$. Let $\varphi$ denote the diffeomorphisms of $M$ with $q \circ \psi=\varphi \circ q$. By differentiating the relation $\psi^{*} \theta=u_{\psi} \cdot \theta$ we get $\varphi^{*} \omega=u_{\psi} \cdot \omega$, hence $\left(\varphi, u_{\psi}\right)$ is an $\omega$-equivariant diffeomorphism.

The proof of the next proposition follows from Remark 8.2 in the Appendix.

Proposition 4.6. For a smooth curve $\psi_{t}$ in $\operatorname{Diff}(P)$ starting at the identity we have $\psi_{t} \in \operatorname{Difff}^{\mathrm{eq}}(P, \theta)^{A} \Leftrightarrow \delta^{l} \psi_{t} \in \mathfrak{X}^{\mathrm{eq}}(P, \theta)^{A} \Leftrightarrow \delta^{r} \psi_{t} \in \mathfrak{X}^{\mathrm{eq}}(P, \theta)^{A}$.

In particular if the flow of an infinitesimal equi-quantomorphism exists, then it consists of equi-quantomorphisms.

Proposition 4.7. The group $\operatorname{Diff}_{A}(P)$ of almost A-equivariant diffeomorphisms contains the group $\operatorname{Diff}^{\mathrm{eq}}(P, \theta)^{A}$ of equi-quantomorphisms as a subgroup. More precisely, the deviation from A-equivariance of an equi-quantomorphism $\psi$ is measured by the isomorphism $u_{\psi} \in \mathrm{GL}(V)$.

Proof. Let $\psi$ be an equi-quantomorphism with $\psi^{*} \theta=u_{\psi} \cdot \theta$. Then $\psi^{-1}$ is an equi-quantomorphism too, with $\left(\psi^{-1}\right)^{*} \theta=\left(u_{\psi}\right)^{-1} \cdot \theta$. For any $a \in A$, the diffeomorphism $\psi \circ \rho(a) \circ \psi^{-1}$ is fiber preserving and $\theta$-invariant, in particular it is of the form $\rho(f)$ with $f \in C^{\infty}(P, A)_{\text {inver }}$ depending on $a$. From Proposition 8.4 in the Appendix, $0=\rho(f)^{*} \theta-\theta=\delta^{l}(f)$, so that $f$ is a constant denoted $\bar{u}(a) \in A$. We obtain that $\psi \circ \rho(a)=\rho(\bar{u}(a)) \circ \psi$, so $\bar{u} \in \operatorname{Aut}(A)$. The infinitesimal version of this identity is $T \psi \circ \dot{\rho}(v)=\dot{\rho}(u(v)) \circ \psi$, where $u \in \mathrm{GL}(V)$ with $\bar{u} \circ \exp =\exp \circ u$.

It remains to be shown that $u=u_{\psi}$. This follows from the above mentioned fact that $\dot{\rho}(v)$ and $\dot{\rho}(u(v))$ are $\psi$-related: $u_{\psi}(v)=i_{\dot{\rho}(v)}\left(\psi^{*} \theta\right)=\psi^{*}\left(i_{\dot{\rho}(u(v))} \theta\right)=u(v)$ for all $v \in V$. 
The group $\mathrm{Hol}^{\mathrm{eq}}(M, \omega)$ of diffeomorphisms preserving the holonomy up to a group automorphism of $A$, called the group of almost holonomy preserving diffeomorphisms is

$\operatorname{Hol}^{\mathrm{eq}}(M, \omega)=\left\{(\varphi, \bar{u}) \in \operatorname{Diff}(M) \times \operatorname{Aut}(A): \forall \ell \in C^{\infty}\left(S^{1}, M\right), h(\varphi \circ \ell)=\bar{u}(h(\ell))\right\}$.

The group $\operatorname{Hol}^{\mathrm{eq}}(M, \omega)$ acts in a natural way on the abelian group $A$. Adapting the idea of the proof of Theorem 2.7 in [11] to the equivariant setting, we will show in the appendix that the group of equi-quantomorphisms is an abelian extension of the group of almost holonomy preserving diffeomorphisms.

Lemma 4.8. Given $(\varphi, \bar{u}) \in \operatorname{Hol}^{\mathrm{eq}}(M, \omega)$ and, for a fixed $x_{0} \in M$, a bijection $\psi_{x_{0}}: q^{-1}\left(x_{0}\right) \rightarrow q^{-1}\left(\varphi\left(x_{0}\right)\right)$ satisfying

$$
\psi_{x_{0}} \circ \rho(a)=\rho(\bar{u}(a)) \circ \psi_{x_{0}}, \quad \forall a \in A,
$$

there exists a unique equi-quantomorphism $\psi$ of $P$ extending $\psi_{x_{0}}$ and descending to the diffeomorphism $\varphi$ of $M$.

Proof. Let $\operatorname{Pt}(c): q^{-1}\left(x_{0}\right) \rightarrow q^{-1}(x)$ denote the parallel transport map along a curve $c$ from $x_{0}$ to $x$ in $M$. It defines a map

$$
\psi_{x}=\operatorname{Pt}(\varphi \circ c) \circ \psi_{x_{0}} \circ \operatorname{Pt}(c)^{-1}: q^{-1}(x) \rightarrow q^{-1}(\varphi(x))
$$

which does not depend on the choice of $c$, because for every loop $\ell$ at $x_{0}$

$$
\operatorname{Pt}(\varphi \circ \ell) \circ \psi_{x_{0}} \circ \operatorname{Pt}(\ell)^{-1}=\rho(\bar{u}(h(\ell))) \circ \psi_{x_{0}} \circ \rho(h(\ell))^{-1} \stackrel{(29)}{=} \psi_{x_{0}} .
$$

The maps $\psi_{x}, x \in M$, glue to a diffeomorphism $\psi$ of $P$ which satisfies $\psi \circ \rho(a)=$ $\rho(\bar{u}(a)) \circ \psi$ for all $a \in A$. Its infinitesimal version is: $\dot{\rho}(v)$ and $\dot{\rho}(u(v))$ are $\psi$-related, where $u \in \mathrm{GL}(V)$ is given by $\exp \circ u=\bar{u} \circ \exp$.

The tangent map $T \psi: T P \rightarrow T P$ maps horizontal vectors to horizontal vectors because for any horizontal lift $c^{\text {hor }}$ of the curve $c$, the curve $\psi \circ c^{\text {hor }}$ is the horizontal lift starting at $\psi\left(c^{\text {hor }}(0)\right)$ of the curve $\varphi \circ c$. Indeed, $\operatorname{Pt}(c)^{-1}\left(c^{\text {hor }}(t)\right)=c^{\text {hor }}(0)$, so by (30) we obtain $\left(\psi \circ c^{\text {hor }}\right)(t)=\operatorname{Pt}\left(\left.\varphi \circ c\right|_{[0, t]}\right)\left(\psi\left(c^{\text {hor }}(0)\right)\right.$. Now one can show that $\psi^{*} \theta=u \cdot \theta$ :

$$
\left(\psi^{*} \theta\right)\left(\eta^{\text {hor }}+\dot{\rho}(v)\right)=\theta\left(T \psi \cdot \eta^{\text {hor }}\right)+\theta(\dot{\rho}(u(v)))=u(v)=(u \cdot \theta)\left(\eta^{\text {hor }}+\dot{\rho}(v)\right)
$$

for all $\eta \in \mathfrak{X}(M)$ and $v \in V$.

Theorem 4.9. The group $\operatorname{Diff}^{\mathrm{eq}}(P, \theta)^{A}$ of equi-quantomorphisms is an abelian extension of the group $\mathrm{Hol}^{\mathrm{eq}}(M, \omega)$ of almost holonomy preserving diffeomorphisms by the natural $\mathrm{Hol}^{\mathrm{eq}}(M, \omega)$-module $A$, i.e.

$$
1 \rightarrow A \stackrel{\rho}{\rightarrow} \operatorname{Diff}^{\mathrm{eq}}(P, \theta)^{A} \stackrel{p}{\rightarrow} \operatorname{Hol}^{\mathrm{eq}}(M, \omega) \rightarrow 1
$$

is an exact sequence of groups. 
Proof. For $\psi \in \operatorname{Diff}^{\text {eq }}(P, \theta)^{A}$ with $\varphi \circ q=q \circ \psi$ and $\psi^{*} \theta=u_{\psi} \cdot \theta$, we define $p(\psi)=\left(q_{*} \psi, \bar{u}_{\psi}\right)$, where $\bar{u}_{\psi} \in \operatorname{Aut}(A)$ with $\bar{u}_{\psi} \circ \exp =\exp \circ u_{\psi}$. In particular $\psi$ is almost $A$-equivariant: $\psi \circ \rho(a)=\rho\left(\bar{u}_{\psi}(a)\right) \circ \psi$ by Proposition 4.7.

We verify that $h(\varphi \circ \ell)=\bar{u}_{\psi}(h(\ell))$ for any $\ell \in C^{\infty}\left(S^{1}, M\right)$, showing that $\varphi$ is an almost holonomy preserving diffeomorphism. First we observe that if $\ell^{\text {hor }}$ is a horizontal lift of the loop $\ell$, then $\psi \circ \ell^{\text {hor }}$ is a horizontal lift of the loop $\varphi \circ \ell$ :

$$
\theta\left(\left(\psi \circ \ell^{\text {hor }}\right)^{\prime}(t)\right)=\theta\left(T \psi \cdot\left(\ell^{\text {hor }}\right)^{\prime}(t)\right)=\left(\psi^{*} \theta\right)\left(\left(\ell^{\text {hor }}\right)^{\prime}(t)\right)=u_{\psi}\left(\theta\left(\left(\ell^{\text {hor }}\right)^{\prime}(t)\right)=0 .\right.
$$

Then the desired identity follows from the computation

$$
\begin{aligned}
\rho\left(\left(\psi \circ \ell^{\text {hor }}\right)(0), h(\varphi \circ \ell)\right) & =\left(\psi \circ \ell^{\text {hor }}\right)(1)=\psi\left(\rho\left(\ell^{\text {hor }}(0), h(\ell)\right)\right) \\
& =\rho\left(\psi\left(\ell^{\text {hor }}(0)\right), \bar{u}_{\psi}(h(\ell))\right) .
\end{aligned}
$$

Thus $p: \operatorname{Diffeq}^{\text {eq }}(P, \theta)^{A} \rightarrow \operatorname{Hol}^{\text {eq }}(M, \omega)$ is well defined. By the previous lemma it is also surjective, and (31) is an exact sequence of groups.

Adapting the proof of Corollary 2.8 in [11] to the equivariant setting, one shows that the identity component $\operatorname{Diff}{ }^{\text {eq }}(P, \theta)_{0}^{A}$ of the group of equi-quantomorphisms is an abelian extension of the group $\operatorname{Diffex}_{\mathrm{ex}}^{\mathrm{eq}}(M, \omega)$ of equi-hamiltonian diffeomorphisms by the natural $\operatorname{Diffex}_{\mathrm{ex}}^{\mathrm{eq}}(M, \omega)-$ module $A$ :

$$
1 \rightarrow A \rightarrow \operatorname{Diff}^{\mathrm{eq}}(P, \theta)_{0}^{A} \rightarrow \operatorname{Diff}_{\mathrm{ex}}^{\mathrm{eq}}(M, \omega) \rightarrow 1 .
$$

We call this the prequantization abelian extension.

Remark 4.10. Like in Remark4.5, if $V=V_{0}$ the closure of the image of $\omega: T M \times_{M}$ $T M \rightarrow V$, the group of equi-hamiltonian diffeomorphisms can be identified with its projection on the first factor. In this case both (31) and (32) can be seen as a restriction of (28).

\section{Group extensions via prequantization}

The results from Sect. 2 concerning central Lie group extensions as pullback of the prequantization extension are generalized in this section to obtain abelian Lie group extensions of $G$ associated to a $G$-equivariant 2 -form $\omega$. Moreover, for simply connected $G$, all its abelian extensions can be obtained in this way.

We consider a smooth action $\lambda$ on $M$ and a linear action $b$ on $V$ such that $\omega$ is $G$-equivariant, i.e. $\lambda_{g}^{*} \omega=b_{g} \cdot \omega$. Let $\dot{\lambda}: \mathfrak{g} \rightarrow \mathfrak{X}(M)$ and $\dot{b}: \mathfrak{g} \rightarrow \mathfrak{g l}(V)$ denote the infinitesimal $\mathfrak{g}$-actions. The pair $(\lambda, b)$ is called an equi-hamiltonian $G$-action if the 1 -form $q^{*} i_{\dot{\lambda}(X)} \omega-\dot{b}(X) \cdot \theta$ is exact for all $X \in \mathfrak{g}$.

In this case $\left(\lambda_{g}, b_{g}\right) \in \operatorname{Diffex}_{\mathrm{ex}}^{\mathrm{eq}}(M, \omega)$ for each $g \in G$, by Proposition 3.9. Then the pull-back of the prequantization abelian extension (32) by $(\lambda, b)$ :

$$
\hat{G}=\left\{(g, \psi) \in G \times \operatorname{Diff}(P): q_{*} \psi=\lambda_{g}, \psi^{*} \theta=b_{g} \cdot \theta\right\},
$$


is an abelian group extension $1 \rightarrow A \stackrel{i}{\rightarrow} \hat{G} \stackrel{p}{\rightarrow} G \rightarrow 1$ with $i(a)=(e, \rho(a))$ and $p(g, \psi)=g$. The induced $G$-module structure on $A$ comes from the linear action $b$ on $V$.

To show that $\hat{G}$ is a Lie group with a smooth action $\hat{\lambda}$ by equi-quantomorphisms of $(P, \theta)$, lifting the action $\lambda$, we use Lemma 3.2 and Lemma 3.3 from [11] and we adapt the proof of Theorem 2.7 from [11] to abelian Lie group extensions. This is resumed in the next theorem.

Theorem 5.1. Given $\omega \in \Omega^{2}(M, V)$ a closed 2-form with discrete period group $\Gamma$ and an equi-hamiltonian $G$-action $(\lambda, b)$, there is an abelian Lie group extension $\hat{G}$ of $G$ by the $G$-module $A=V / \Gamma$, integrating the $V$-valued Lie algebra 2-cocycle on $\mathfrak{g}$

$$
(X, Y) \mapsto-\omega(\dot{\lambda}(X), \dot{\lambda}(Y))\left(x_{0}\right),
$$

whose cohomology class does not depend on the choice of the point $x_{0} \in M$. There is also a $\hat{G}$-action $\hat{\lambda}$ on $P$, lifting the $G$-action $\lambda$, and such that $\theta$ is $\hat{G}$-equivariant, i.e. $\hat{\lambda}_{\hat{g}}^{*} \theta=b_{p(\hat{g})} \cdot \theta$ for any $\hat{g} \in \hat{G}$.

Proof. Let $\lambda^{x_{0}}: g \in G \mapsto \lambda_{g}\left(x_{0}\right) \in M$ be the orbit map, and let $\left(\lambda^{x_{0}}\right)^{*} P \rightarrow G$ be the pull-back of the $A$-bundle $P \rightarrow M$. From Lemma 3.2 in [11] follows that an element $y_{0} \in P$ with $q\left(y_{0}\right)=x_{0}$ defines a bijection $(g, \psi) \in \hat{G} \mapsto\left(g, \psi\left(y_{0}\right)\right) \in$ $\left(\lambda^{x_{0}}\right)^{*} P$, and the smooth manifold structure transported on $\hat{G}$ by this bijection does not depend on the choice of $x_{0}$.

We define the $\hat{G}$-action $\hat{\lambda}$ on $P$ by

$$
\hat{\lambda}:((g, \psi), y) \in \hat{G} \times P \mapsto \psi(y) \in P .
$$

It lifts the $G$-action $\lambda: G \times M \rightarrow M$ and $\theta$ is $\hat{G}$-equivariant because $\psi$ is an equi-quantomorphism. Its restriction to $A$ is $\hat{\lambda}_{a}=\rho(a)$ for $a \in A$.

In order to show that $\hat{\lambda}$ is smooth, we put product coordinates on $P$ and $\hat{G}$. Any smooth local section $s_{M}: U \subset M \rightarrow P$ of $q$, with $s_{M}\left(x_{0}\right)=y_{0}$, defines a local smooth section $s_{G}(g)=\left(g, \psi_{g}\right) \in \hat{G}$ of $p$, for $g$ sufficiently close to the identity of $G$ in order to have $\lambda_{g}\left(x_{0}\right) \in U$. Here $\psi_{g}$ is uniquely defined by the condition $\psi_{g}\left(y_{0}\right)=s_{M}\left(\lambda_{g}\left(x_{0}\right)\right)$.

We fix an open neighbourhood $U_{G}$ of $e$ and a neighbourhood $U_{M}$ of $x$ diffeomorphic to an open convex subset of the modeling space, such that $U_{G} \cdot U_{M} \subset U$. Then we define a function $f: U_{G} \times U_{M} \rightarrow A$ with $f\left(g, x_{0}\right)=1$ for all $g \in U_{G}$ by the relation

$$
\psi_{g}\left(s_{M}(x)\right)=\rho\left(s_{M}\left(\lambda_{g}(x)\right), f(g, x)\right) .
$$

In other words, for $g \in U_{G}$ the expression of $\psi_{g}$ in product coordinates is $\psi_{g}$ : $(x, a) \in U_{M} \times A \mapsto\left(\lambda_{g}(x), f(g, x) \bar{b}_{g}(a)\right) \in U \times A$, where $\bar{b}_{g} \in \operatorname{Aut}(A)$ with $\bar{b}_{g} \circ \exp =\exp \circ b_{g}$. This can be deduced from

$$
\left(\psi_{g} \circ \rho(a)\right)\left(s_{M}(x)\right)=\left(\rho\left(\bar{b}_{g}(a)\right) \circ \psi_{g}\right)\left(s_{M}(x)\right)=\rho\left(f(g, x) \bar{b}_{g}(a)\right)\left(s_{M}\left(\lambda_{g}(x)\right)\right) .
$$


The connection 1 -form in product coordinates $U \times A$ is $\theta=q^{*} \alpha+q_{A}^{*} \theta_{A}^{l}$, with $q_{A}: q^{-1}(U) \rightarrow A$ the second projection, $\alpha=s_{M}^{*} \theta \in \Omega^{1}(U, V)$ and $\theta_{A}^{l}=\delta^{l}\left(1_{A}\right) \in$ $\Omega^{1}(A, V)$ the Maurer-Cartan form on $A$. In particular $d \alpha=\omega$ on $U \subset M$. The condition $\psi_{g}^{*} \theta=b_{g} \cdot \theta$ implies $\delta^{l}\left(f_{g}\right)=\lambda_{g}^{*} \alpha-b_{g} \cdot \alpha$. Indeed,

$$
\begin{aligned}
0=\psi_{g}^{*}\left(q^{*} \alpha\right. & \left.+q_{A}^{*} \theta_{A}^{l}\right)-b_{g} \cdot\left(q^{*} \alpha+q_{A}^{*} \theta_{A}^{l}\right)=q^{*}\left(\lambda_{g}^{*} \alpha-b_{g} \cdot \alpha\right) \\
& +\delta^{l}\left(m \circ\left(\bar{b}_{g} \circ q_{A}, f_{g} \circ q\right)\right)-q_{A}^{*} \delta^{l}\left(\bar{b}_{g}\right)=q^{*}\left(\lambda_{g}^{*} \alpha-b_{g} \cdot \alpha-\delta^{l}\left(f_{g}\right)\right)
\end{aligned}
$$

because $q_{A} \circ \psi_{g}=m \circ\left(\bar{b}_{g} \circ q_{A}, f_{g} \circ q\right)$ for $m$ the group multiplication map of $A$ and $\delta^{l}\left(m \circ\left(h_{1}, h_{2}\right)\right)=\delta^{l} h_{1}+\delta^{l} h_{2}$ for $h_{1}, h_{2}: P \rightarrow A$.

Using the Poincaré Lemma applied to the closed 1-form $\lambda_{g}^{*} \alpha-b_{g} \cdot \alpha$ on the convex set $U_{M}$, we obtain that $f$ is a smooth function. Then

$$
\left(\rho(a) \circ \psi_{g}\right)\left(\rho\left(s_{M}(x), a^{\prime}\right)\right)=\rho\left(s_{M}\left(\lambda_{g}(x)\right), f(g, x) a \bar{b}_{g}\left(a^{\prime}\right)\right)
$$

assures the following expression of $\hat{\lambda}$ in product coordinates:

$$
\hat{\lambda}\left((g, a),\left(x, a^{\prime}\right)\right)=\left(\lambda_{g}(x), f(g, x) a \bar{b}_{g}\left(a^{\prime}\right)\right),
$$

thus showing the smoothness of $\hat{\lambda}$.

The $A$-valued local group 2-cocycle on $G$ corresponding to the section $s_{G}$ is

$$
c\left(g_{1}, g_{2}\right)=f\left(g_{1}, \lambda_{g_{2}}\left(x_{0}\right)\right)
$$

because

$$
\begin{aligned}
\psi_{g_{1}} \psi_{g_{2}}\left(y_{0}\right) & =\psi_{g_{1}}\left(s_{M}\left(\lambda_{g_{2}}\left(x_{0}\right)\right)\right) \stackrel{\text { 355 }}{=} \rho\left(s_{M}\left(\lambda_{g_{1} g_{2}}\left(x_{0}\right)\right), f\left(g_{1}, \lambda_{g_{2}}\left(x_{0}\right)\right)\right) \\
& =\rho\left(\psi_{g_{1} g_{2}}\left(y_{0}\right), f\left(g_{1}, \lambda_{g_{2}}\left(x_{0}\right)\right)\right) .
\end{aligned}
$$

This shows the smoothness of multiplication and inversion in an identity neighborhood in $\hat{G}$. That the left multiplications are smooth, follows from the fact that $\hat{G}$ acts by smooth maps on the bundle $P$. Now Lemma 3.3 in [1] implies that $\hat{G}$ is a Lie group.

The corresponding Lie algebra extension $0 \rightarrow V \rightarrow \hat{\mathfrak{g}} \rightarrow \mathfrak{g} \rightarrow 0$ is the pullback by $(\dot{\lambda}, \dot{b}): \mathfrak{g} \rightarrow \mathfrak{X}_{\mathrm{ex}}^{\mathrm{eq}}(M, \omega)$ of the abelian extension (27), hence a defining Lie algebra 2 -cocycle is (34), the pull-back by $(\dot{\lambda}, \dot{b})$ of the 2-cocycle from Theorem 4.4.

Theorem 5.2. Every abelian Lie group extension $1 \rightarrow A=V / \Gamma \rightarrow \hat{G} \stackrel{q}{\rightarrow} G \rightarrow 1$ of a simply connected regular Lie group $G$ can be obtained as a pull-back of the prequantization abelian extension (32).

Proof. Let $0 \rightarrow V \rightarrow \hat{\mathfrak{g}} \stackrel{q}{\rightarrow} \mathfrak{g} \rightarrow 0$ be the abelian Lie algebra extension corresponding to the given Lie group extension, and let $s: \mathfrak{g} \rightarrow \hat{\mathfrak{g}}$ be a continuous section. We 
denote by $\sigma$ the $V$-valued 2-cocycle on $\mathfrak{g}$ defined by $s$, and by $p: \hat{\mathfrak{g}} \rightarrow V$ the associated projection, so $\sigma(X, Y)=[s(X), s(Y)]-s([X, Y])$ and $p(\hat{X})=\hat{X}-s(q(\hat{X}))$. We use the identification $\hat{X}=(X, v)$ for $X=q(\hat{X})$ and $v=p(\hat{X})$ (in this proof the same letter $q$ denotes both projections $\hat{G} \rightarrow G$ and $\hat{\mathfrak{g}} \rightarrow \mathfrak{g})$.

Let $\lambda$ be the left translation on $G$ and $b$ the linear $G$-action on $V$ induced by the $G$-module structure of $A$ determined by the abelian extension of $G$ by $A$, namely $g \cdot a=\hat{g} a \hat{g}^{-1}$ for any $\hat{g} \in \hat{G}$ with $q(\hat{g})=g$. With respect to these two $G$-actions, the $G$-equivariant 2-form $\omega:=-\sigma^{\text {eq }} \in \Omega^{2}(G, V)$ is closed, because $\sigma$ is a 2-cocycle. Since $\lambda_{g}^{*} \omega=b_{g} \cdot \omega$, we obtain a group homomorphism $(\lambda, b): G \rightarrow \operatorname{Diffeq}^{\text {eq }}(G, \omega)$. We wish to have a group homomorphism into $\operatorname{Diffex}_{\mathrm{ex}}^{\mathrm{eq}}(G, \omega)$, and for this we need a principal connection on a principal bundle over $G$ with curvature $\omega$.

The abelian extension $\hat{G}$ is a principal $A$-bundle over $G$. Here we need the principal $A$-action on $\hat{G}$ to be given by left translations, hence $\rho$ is a left action: $\rho(a) \hat{g}=a \hat{g}$. We show that the $\hat{G}$-equivariant 1 -form $\theta=p^{\text {eq }} \in \Omega^{1}(\hat{G}, V)$ (in the sense that $\hat{\lambda}_{\hat{g}}^{*} \theta=b_{g} \cdot \theta$ where $\hat{\lambda}$ denotes left translation on $\hat{G}$ ) with identity value $\theta_{e}=p$, is a principal connection. For this we only have to verify the identity $\theta(\dot{\rho}(v))=v$ for all $v \in V$, because the $A$-invariance $\rho(a)^{*} \theta=\theta$ for all $a \in A$ is a special case of the $\hat{G}$-equivariance:

$$
\theta(\dot{\rho}(v))(\hat{g})=\theta\left(\left.\frac{d}{d t}\right|_{0}(\exp t v) \hat{g}\right)=p^{\mathrm{eq}}\left(\hat{g} \cdot \operatorname{Ad}_{\hat{g}^{-1}}(v)\right)=b_{g} \cdot p\left(b_{g^{-1}}(v)\right)=v \text {. }
$$

The curvature of the principal connection $\theta$ is $\omega$, because $p\left(\left[\left(X_{1}, v_{1}\right),\left(X_{2}, v_{2}\right)\right]\right)=$ $\dot{b}\left(X_{1}\right) v_{2}-\dot{b}\left(X_{2}\right) v_{1}+\sigma\left(X_{1}, X_{2}\right)$ implies that the Chevalley-Eilenberg differential of the $V$-valued 1 -cochain $p$ on $\hat{\mathfrak{g}}$ is the pull-back of $-\sigma$ to $\hat{\mathfrak{g}}$, so $d \theta=d p^{\text {eq }}=$ $-q^{*} \sigma^{\mathrm{eq}}=q^{*} \omega$.

Next we show that the $G$-action $(\lambda, b)$ is equi-hamiltonian, i.e. the infinitesimal flux cocycle flux ${ }^{\mathrm{eq}}$ vanishes on $(\dot{\lambda}(Y), \dot{b}(Y))=\left(Y^{r}, \dot{b}(Y)\right) \in \mathfrak{X}^{\mathrm{eq}}(G, \omega)$ for all $Y \in \mathfrak{g}$, where $Y^{r}$ denotes the right invariant vector field on $G$ with identity value $Y^{r}(e)=$ $Y$ :

$$
\operatorname{flux}^{\mathrm{eq}}\left(Y^{r}, \dot{b}(Y)\right)=\left[q^{*} i_{Y^{r}} \omega-\dot{b}(Y) \cdot \theta\right]=\left[q^{*} i_{Y^{r}} \sigma^{\mathrm{eq}}+\dot{b}(Y) \cdot p^{\mathrm{eq}}\right] \in H^{1}(\hat{G}, V) .
$$

We will use Proposition 8.5 from the Appendix to show that the 1 -form $q^{*} i_{Y^{r}} \sigma^{\mathrm{eq}}+$ $\dot{b}(Y) \cdot p^{\text {eq }}$ on $\hat{G}$ is exact, namely we will show that it is the equivariant 1 -form coming from a Lie algebra 1-cocycle which can be integrated to a group 1-cocycle.

There is a group 1-cocycle $\kappa: \hat{G} \rightarrow \operatorname{Lin}(\mathfrak{g}, V)$ involved in the expression of the adjoint action on the abelian extension $\hat{G}[10]$ :

$$
\operatorname{Ad}_{\hat{g}}(Y, w)=\left(\operatorname{Ad}_{g} Y, b_{g}(w)-\kappa(\hat{g})\left(\operatorname{Ad}_{g} Y\right)\right)
$$

From the expression of the Lie bracket in $\hat{\mathfrak{g}}$ :

$$
\operatorname{ad}((X, v))(Y, w)=[(X, v),(Y, w)]=([X, Y], \dot{b}(X) w-\dot{b}(Y) v+\sigma(X, Y)
$$


we deduce that the Lie algebra 1 -cocycle $\alpha: \hat{\mathfrak{g}} \rightarrow \operatorname{Lin}(\mathfrak{g}, V)$ corresponding to $\kappa$ is $\alpha(X, v)(Y)=\dot{b}(Y) v-\sigma(X, Y)$. With the notations $\dot{b}\llcorner: v \in V \mapsto \dot{b}(\cdot) v \in \operatorname{Lin}(\mathfrak{g}, V)$ and $\sigma^{\llcorner}: X \in \mathfrak{g} \mapsto i_{X} \sigma \in \operatorname{Lin}(\mathfrak{g}, V)$, we write

$$
\alpha=\dot{b}^{\llcorner} \circ p-\sigma^{`} \circ q \text {. }
$$

To express the terms of (37) we need the identities:

$$
\dot{b}(Y) \cdot p^{\mathrm{eq}}=\mathrm{ev}_{Y} \circ\left(\dot{b}^{\llcorner} \circ p\right)^{\mathrm{eq}} \text { and } q^{*} i_{Y^{r}} \sigma^{\mathrm{eq}}=-\mathrm{ev}_{Y} \circ\left(\sigma^{\llcorner} \circ q\right)^{\mathrm{eq}},
$$

where $\operatorname{ev}_{Y}: \operatorname{Lin}(\mathfrak{g}, V) \rightarrow V$ for $Y \in \mathfrak{g}$ denotes the evaluation map. Indeed,

$$
\begin{aligned}
\dot{b}(Y) \cdot p^{\mathrm{eq}}(\hat{g} \cdot \hat{X}) & =\dot{b}(Y)\left(b_{g} \cdot p(\hat{X})\right)=b_{g} \cdot \dot{b}\left(\operatorname{Ad}_{g^{-1}} Y\right) p(\hat{X})=b_{g} \cdot \dot{b}(p(\hat{X}))\left(\operatorname{Ad}_{g^{-1}} Y\right) \\
& =\left(g \cdot\left(\dot{b}^{\llcorner} \circ p\right)(\hat{X})\right)(Y)=\operatorname{ev}_{Y} \circ\left(\dot{b}^{\llcorner} \circ p\right)^{\mathrm{eq}}(\hat{g} \cdot \hat{X})
\end{aligned}
$$

and

$$
\begin{gathered}
q^{*} i_{Y^{r}} \sigma^{\mathrm{eq}}(\hat{g} \cdot \hat{X})=\sigma^{\mathrm{eq}}(Y \cdot g, g \cdot X)=b_{g} \cdot \sigma\left(\operatorname{Ad}_{g^{-1}} Y, X\right)=-b_{g} \cdot\left(\sigma^{\smile}(X)\right)\left(\operatorname{Ad}_{g^{-1}} Y\right) \\
=-\left(g \cdot\left(\sigma^{`} \circ q(\hat{X})\right)\right)(Y)=-\operatorname{ev}_{Y} \circ\left(\sigma^{\llcorner} \circ q\right)^{\mathrm{eq}}(\hat{g} \cdot \hat{X}) .
\end{gathered}
$$

We continue the computation (37):

$$
\operatorname{flux}^{\mathrm{eq}}\left(Y^{r}, \dot{b}(Y)\right)=\mathrm{ev}_{Y} \circ\left[-\left(\sigma^{\llcorner} \circ q\right)^{\mathrm{eq}}+\left(\dot{b}^{\llcorner} \circ p\right)^{\mathrm{eq}}\right] \stackrel{(38)}{=} \mathrm{ev}_{Y} \circ\left[\alpha^{\mathrm{eq}}\right]=\mathrm{ev}_{Y} \circ[d \kappa]=0
$$

Knowing now that $(\lambda, b)$ is an equi-hamiltonian action, we pull-back the prequantization abelian extension

$$
1 \rightarrow A \rightarrow \operatorname{Diff}^{\mathrm{eq}}\left(\hat{G}, \theta=p^{\mathrm{eq}}\right)_{0}^{A} \rightarrow \operatorname{Diffex}_{\mathrm{ex}}^{\mathrm{eq}}\left(G, \omega=-\sigma^{\mathrm{eq}}\right) \rightarrow 1
$$

by the equi-hamiltonian action $(\lambda, b)$. The result is an abelian Lie group extension of $G$ by $A$, integrating the Lie algebra 2-cocycle $\sigma$ because

$$
-\omega(\dot{\lambda}(X), \dot{\lambda}(Y))(e)=\sigma^{\mathrm{eq}}\left(X^{r}, Y^{r}\right)(e)=\sigma(X, Y), \quad X, Y \in \mathfrak{g} .
$$

It is isomorphic with the given extension $\hat{G}$ since they have the same Lie algebras and $G$ is simply connected and regular [10] Chapter VIII.

\section{Group 2-cocycle}

In the case of an exact 2-form $\omega=d \alpha$ for some $\alpha \in \Omega^{1}(M, V)$, the principal bundle with curvature $\omega$ is the trivial bundle $P=M \times V \stackrel{q}{\rightarrow} M$ with principal connection 1 -form $\theta=q^{*} \alpha+\theta_{V}$, where $\theta_{V}$ denotes the Maurer-Cartan form on $V$. Then the prequantization central extension (14) for $(M, d \alpha)$ is defined by $V$-valued group 2 -cocycles on the hamiltonian group $\operatorname{Diff}_{\mathrm{ex}}(M, \omega)$ :

$$
c\left(\varphi_{1}, \varphi_{2}\right)=f\left(\varphi_{2}\right)(x)-f\left(\varphi_{1} \varphi_{2}\right)(x)+f\left(\varphi_{1}\right)\left(\varphi_{2}(x)\right)
$$


where $f: \operatorname{Diff}_{\mathrm{ex}}(M, \omega) \rightarrow C^{\infty}(M, V)$ is a map satisfying $f\left(1_{M}\right)=0$ and $d(f(\varphi))=$ $\alpha-\varphi^{*} \alpha$. Here $x \in M$ is arbitrary: $c\left(\varphi_{1}, \varphi_{2}\right)$ does not depend on $x$. The cocycle $c$ is cohomologous to the following group cocycle on $\operatorname{Diff}_{\mathrm{ex}}(M, \omega)$ :

$$
B\left(\varphi_{1}, \varphi_{2}\right)=\int_{x}^{\varphi_{2}(x)}\left(\alpha-\varphi_{1}^{*} \alpha\right)
$$

In the special case when $H^{1}(M, \mathbb{R})=0$, the cocycle $B$ can be extended to the group $\operatorname{Diff}(M, \omega)$ of $\omega$-preserving diffeomorphisms [6]. The same holds for the cocycle $c$. This follows from the fact that, for any $\varphi \in \operatorname{Diff}(M, \omega)$, the 1 -form $\varphi^{*} \alpha-\alpha$ is closed, hence exact.

We show that the prequantization abelian extension (32) is described by a similar $V$-valued group 2-cocycle on $\operatorname{Diff}_{\mathrm{ex}}^{\mathrm{eq}}(M, \omega)$.

Theorem 6.1. Given $\omega=d \alpha$ for $\alpha \in \Omega^{1}(M, V)$, the identity component of the group of equi-quantomorphisms $\operatorname{Diff}^{\mathrm{eq}}(P, \theta)_{0}^{A}$ is the abelian extension of the group of equi-hamiltonian diffeomorphisms $\operatorname{Diff}_{\mathrm{ex}}^{\mathrm{eq}}(M, \omega)$ by $V$ with cohomology class defined by the $V$-valued group 2-cocycle c on $\operatorname{Diff}_{\mathrm{ex}}^{\mathrm{eq}}(M, \omega)$,

$$
c\left(\left(\varphi_{1}, u_{1}\right),\left(\varphi_{2}, u_{2}\right)\right)=u_{1}\left(f\left(\varphi_{2}, u_{2}\right)(x)\right)-f\left(\varphi_{1} \varphi_{2}, u_{1} u_{2}\right)(x)+f\left(\varphi_{1}, u_{1}\right)\left(\varphi_{2}(x)\right),
$$

where the map $f: \operatorname{Diffex}_{\mathrm{ex}}^{\mathrm{eq}}(M, \omega) \rightarrow C^{\infty}(M, V)$ is determined by $f\left(1_{M}, 1_{V}\right)=0$ and $d(f(\varphi, u))=u \cdot \alpha-\varphi^{*} \alpha$. Different choices for $f$ define cohomologous cocycles. The cocycle $c$ is cohomologous to the group cocycle

$$
B\left(\left(\varphi_{1}, u_{1}\right),\left(\varphi_{2}, u_{2}\right)\right)=\int_{x}^{\varphi_{2}(x)}\left(u_{1} \cdot \alpha-\varphi_{1}^{*} \alpha\right) .
$$

Proof. The existence of the map $f$ follows from Remark 3.10.

An arbitrary equi-quantomorphism $\psi$ of $\left(P=M \times V, \theta=q^{*} \alpha+\theta_{V}\right)$ is of the form $\psi(x, v)=\left(\varphi(x), m_{\psi}(x, v)\right)$, where $\varphi=q_{*} \psi$ and $m_{\psi}: M \times V \rightarrow V$. The condition $\psi^{*} \theta=u \cdot \theta$ becomes $d m_{\psi}=d(f(\varphi, u)+u)$, since $\psi^{*}\left(\theta_{V}\right)=d m_{\psi}$ and $\psi^{*} q^{*} \alpha=q^{*} \varphi^{*} \alpha=q^{*}(u \cdot \alpha-d(f(\varphi, u)))$. Hence the map $m_{\psi}$ is of the form $m_{\psi}(x, v)=f(\varphi, u)(x)+u(v)+a$ for some $a \in V$ so

$$
\psi(x, v)=(\varphi(x), f(\varphi, u)(x)+u(v)+a) .
$$

In this way we get a bijection $\psi \mapsto((\varphi, u), a)$ between $\operatorname{Diffeq}^{e}(P, \theta)^{A}$ and the cartesian product $\operatorname{Diffex}_{\mathrm{ex}}^{\mathrm{eq}}(M, \omega) \times V \subset \operatorname{Diff}(M) \times \mathrm{GL}(V) \times V$. The following computation shows that the group $\operatorname{Diff}^{\mathrm{eq}}(P, \theta)_{0}^{A}$ is isomorphic to the abelian extension defined by the given $V$-valued group 2-cocycle $c$ on $\operatorname{Diff}_{\mathrm{ex}}^{\mathrm{eq}}(M, \omega)$ :

$$
\begin{aligned}
& \left(\varphi_{1}, u_{1}, a_{1}\right) \circ\left(\varphi_{2}, u_{2}, a_{2}\right)(x, v)=\left(\varphi_{1}, u_{1}, a_{1}\right)\left(\varphi_{2}(x), f\left(\varphi_{2}, u_{2}\right)(x)+u_{2}(v)+a_{2}\right) \\
& =\left(\varphi_{1} \varphi_{2}(x), f\left(\varphi_{1}, u_{1}\right)\left(\varphi_{2}(x)\right)+u_{1} f\left(\varphi_{2}, u_{2}\right)(x)+u_{1} u_{2}(v)+u_{1}\left(a_{2}\right)+a_{1}\right) \\
& =\left(\varphi_{1} \varphi_{2}(x), f\left(\varphi_{1} \varphi_{2}, u_{1} u_{2}\right)(x)+u_{1} u_{2}(v)+a_{1}+u_{1}\left(a_{2}\right)+c\left(\left(\varphi_{1}, u_{1}\right),\left(\varphi_{2}, u_{2}\right)\right)\right) \\
& =\left(\varphi_{1} \varphi_{2}, u_{1} u_{2}, a_{1}+u_{1}\left(a_{2}\right)+c\left(\left(\varphi_{1}, u_{1}\right),\left(\varphi_{2}, u_{2}\right)\right)\right)(x, v)
\end{aligned}
$$


for all $(x, v) \in P$, which means $\left(\left(\varphi_{1}, u_{1}\right), a_{1}\right) \circ\left(\left(\varphi_{2}, u_{2}\right), a_{2}\right)=\left(\left(\varphi_{1}, u_{1}\right)\left(\varphi_{2}, u_{2}\right), a_{1}+\right.$ $\left.\left(\varphi_{1}, u_{1}\right) \cdot a_{2}+c\left(\left(\varphi_{1}, u_{1}\right),\left(\varphi_{2}, u_{2}\right)\right)\right)$.

The cocycles $c$ and $B$ differ by the coboundary of the 1-cochain $(\varphi, u) \mapsto$ $f(\varphi, u)(x)$ on Diffex $(M, \omega)$, so they are cohomologous and both describe the prequantization abelian extension.

Remark 6.2. In the special case when $H^{1}(M, V)=0$, both cocycles $B$ and $c$ from Theorem 6.1 can be extended to the group $\operatorname{Diff}^{\text {eq }}(M, \omega)$ of $\omega$-equivariant diffeomorphisms. This follows from the fact that, for any $\varphi \in \operatorname{Diffe}^{\mathrm{eq}}(M, \omega)$, the $V$-valued 1-form $\varphi^{*} \alpha-u \cdot \alpha$ on $M$ is closed, hence exact.

Remark 6.3. Given an exact 2 -form $\omega=d \alpha \in \Omega^{2}(M, V)$ and an equi-hamiltonian $G$-action $(\lambda, b)$, Theorem 5.1 provides an abelian Lie group extension $\hat{G}$ of $G$ by the $G$-module $V$, which can be defined also by a group 2-cocycle, the pull-back of the cocycle from Theorem 6.1 by the group homomorphism $(\lambda, b): G \rightarrow \operatorname{Diffex}_{\mathrm{ex}}^{\mathrm{eq}}(M, \omega)$ :

$$
c\left(g_{1}, g_{2}\right)=b_{g_{1}}\left(f\left(g_{2}\right)(x)\right)-f\left(g_{1} g_{2}\right)(x)+f\left(g_{1}\right)\left(\lambda_{g_{2}}(x)\right),
$$

where $f(g) \in C^{\infty}(M, V)$ with $f(e)=0$ and $d(f(g))=b_{g} \cdot \alpha-\lambda_{g}^{*} \alpha$, and $x \in M$ arbitrary. The $\hat{G}$-action on $P=M \times V$ lifting the $G$-action and such that $\theta$ is $\hat{G}$-equivariant is $(g, v) \cdot\left(x, v^{\prime}\right)=\left(\lambda_{g}(x), f(g)(x)+b_{g}\left(v^{\prime}\right)+v\right)$.

\section{$7 \quad$ Examples}

Theorem 5.1 provides a geometric construction of several abelian Lie group extensions of diffeomorphism groups. In the examples below, the manifold $M$ will always be a homogeneous manifold $G / H$, with $H$ a connected Lie subgroup of $G$ and $V$ a $G$-module. The $G$-equivariant closed 2 -form $\omega$ on $G / H$ is uniquely defined by a $V$-valued Lie algebra 2-cocycle $\sigma$ on $\mathfrak{g}$ satisfying two properties:

1. The kernel of $\sigma$ contains the Lie algebra $\mathfrak{h}$ of $H$, so that $\sigma$ descends to a skew-symmetric bilinear form on $\mathfrak{g} / \mathfrak{h}$.

2. $\sigma$ is $H$-equivariant, i.e. $\sigma(\operatorname{Ad}(g) X, \operatorname{Ad}(g) Y)=b(g) \cdot \sigma(X, Y)$ for all $X, Y \in$ $\mathfrak{g}$ and $g \in H$. The subgroup $H$ being connected, the last condition is equivalent to $\sigma([Z, X], Y)+\sigma(X,[Z, Y])=\dot{b}(Z) \cdot \sigma(X, Y)$ for all $X, Y \in \mathfrak{g}$ and $Z \in \mathfrak{h}$.

Example 7.1. We consider the group Diff $+\left(S^{1}\right)$ of orientation preserving diffeomorphisms of the circle and its right modules $\mathfrak{F}_{\lambda}$ of $\lambda$-densities on the circle: $b_{\lambda}(\varphi) f=\left(\varphi^{\prime}\right)^{\lambda}(f \circ \varphi)$ for $\varphi \in \operatorname{Diff}_{+}\left(S^{1}\right)$ and $f \in C^{\infty}\left(S^{1}\right)$. The $\mathfrak{X}\left(S^{1}\right)$-module structure on $\mathfrak{F}_{\lambda}$ is given by $\dot{b}_{\lambda}(X) f=X f^{\prime}+\lambda X^{\prime} f$ for $X \in \mathfrak{X}\left(S^{1}\right)$. Here we identify $\lambda$-densities $f(x)(d x)^{\lambda}$ and vector fields $X(x) \frac{d}{d x}$ with smooth functions $f$ and $X$ on the circle. 
The abelian extensions of $\mathfrak{X}\left(S^{1}\right)$ defined with the $\mathfrak{F}_{\lambda}-$ valued cocycles

$$
\begin{gathered}
\sigma_{0}(X, Y)=\int_{0}^{1}\left(X^{\prime} Y^{\prime \prime}-X^{\prime \prime} Y^{\prime}\right) d x \in \mathbb{R} \subset \mathfrak{F}_{0} \\
\sigma_{1}(X, Y)=X^{\prime} Y^{\prime \prime}-X^{\prime \prime} Y^{\prime} \in \mathfrak{F}_{1} \\
\sigma_{2}(X, Y)=X^{\prime} Y^{\prime \prime \prime}-X^{\prime \prime \prime} Y^{\prime} \in \mathfrak{F}_{2}
\end{gathered}
$$

integrate to abelian extensions of $\operatorname{Diff}_{+}\left(S^{1}\right)$. Corresponding group cocycles are presented in [13. These abelian extensions can also be obtained geometrically by the Theorem [5.1, taking $M$ to be the contractible homogeneous space Diff $+\left(S^{1}\right) / S^{1}$, where $S^{1}$ is identified with the subgroup of rotations of $S^{1}$. The existence of the Diff $+\left(S^{1}\right)$-equivariant $\mathfrak{F}_{\lambda}$-valued closed 2 -form $\omega_{\lambda}$ on $M$ defined by the 2-cocycle $\sigma_{\lambda}$ for $\lambda=0,1,2$ is ensured by the $S^{1}$-equivariance of $\sigma_{\lambda}$ and the fact that the constant vector fields belong to the kernel of $\sigma_{\lambda}$.

The abelian extensions of $\mathfrak{X}\left(S^{1}\right)$ by $\mathfrak{F}_{\lambda}$ defined with the 2-cocycles

$$
\begin{gathered}
\bar{\sigma}_{0}(X, Y)=X Y^{\prime}-X^{\prime} Y \in \mathfrak{F}_{0} \\
\bar{\sigma}_{1}(X, Y)=X Y^{\prime \prime}-X^{\prime \prime} Y \in \mathfrak{F}_{1} \\
\bar{\sigma}_{2}(X, Y)=X Y^{\prime \prime \prime}-X^{\prime \prime \prime} Y \in \mathfrak{F}_{2}
\end{gathered}
$$

integrate to abelian extensions of the universal covering group $\widetilde{\operatorname{Diff}}_{+}\left(S^{1}\right)$ [10] Section 10. For the geometric construction of these abelian extensions, in Theorem 5.1 we take $G=\widetilde{\operatorname{Diff}}_{+}\left(S^{1}\right)$ acting by left translations on $M=\widetilde{\operatorname{Diff}}_{+}\left(S^{1}\right)$, which is contractible. The $\widetilde{\operatorname{Diff}}+\left(S^{1}\right)$-equivariant $\mathfrak{F}_{\lambda}$-valued closed 2 -form $\bar{\omega}_{\lambda}$ is uniquely defined by its value $\bar{\sigma}_{\lambda}$ at the identity, for $\lambda=0,1,2$.

Example 7.2. Given a volume form $\mu$ on $M$, a non-trivial $\Omega^{1}(M) / d \Omega^{0}(M)$-valued Lie algebra 2-cocycle on $\mathfrak{X}(M)$ is $\sigma(X, Y)=(\operatorname{div} X) d(\operatorname{div} Y)$. A group 2-cocycle on $\operatorname{Diff}(M)$ integrating $\sigma$ is constructed in [2].

For the geometric construction of an abelian Lie group extension of $\operatorname{Diff}(M)$ by its module $\Omega^{1}(M) / d \Omega^{0}(M)$, we remark that the 2 -cocycle $\sigma$ is $\operatorname{Diff}(M, \mu)$ equivariant and the Lie algebra $\mathfrak{X}(M, \mu)$ of divergence free vector fields is contained in the kernel of $\sigma$, hence there is a closed $\operatorname{Diff}(M)$-equivariant 2 -form $\omega$ on the homogeneous space $\operatorname{Diff}(M) / \operatorname{Diff}(M, \mu)$. By a result of Moser [8], this space can be identified with the contractible space of all volume forms of total mass 1 . Now Theorem 5.1 can be applied to $\omega$.

Example 7.3. Let $\theta$ be a connection 1 -form on the principal GL $(n, \mathbb{R})$-bundle of frames $\pi: P(M) \rightarrow M$. Gelfand's cocycle presented in [15] is the $\Omega^{2}(M)$-valued 2-cocycle $\sigma$ on $\mathfrak{X}(M)$ defined by $\pi^{*} \sigma(X, Y)=\operatorname{tr}\left(L_{\tilde{X}} \theta \wedge L_{\tilde{Y}} \theta\right)$, where $\tilde{X}, \tilde{Y} \in$ $\mathfrak{X}(P(M))$ are canonical lifts of $X, Y \in \mathfrak{X}(M)$. In the special case $M=\mathbb{T}^{n}$ the $n$-torus, a group 2-cocycle on Diff $\left(\mathbb{T}^{n}\right)$ integrating $\sigma$ is constructed in [2].

For the 2-sphere $S^{2}$, Theorem 5.1 provides a geometric construction of an abelian Lie group extension of $\operatorname{Diff}\left(S^{2}\right)$ by $\Omega^{2}\left(S^{2}\right)$ integrating Gelfand's cocycle. 
We choose the connection $\theta$ on the principal bundle of frames of $S^{2}$ induced by the canonical Riemannian metric on $S^{2}$. The connected component of the isometry group of $S^{2}$ is $\mathrm{SO}(3) \subset \operatorname{Diff}\left(S^{2}\right)$, so $L_{\tilde{X}} \theta=0$ for $X \in \mathfrak{s o}(3) \subset \mathfrak{X}\left(S^{2}\right)$. The 2-cocycle $\sigma$ is $\mathrm{SO}(3)$-equivariant and the Lie algebra $\mathfrak{s o}(3)$ is contained in the kernel of $\sigma$, hence there exists a closed $\operatorname{Diff}\left(S^{2}\right)$-equivariant 2-form $\omega$ on the homogeneous space $\operatorname{Diff}\left(S^{2}\right) / \mathrm{SO}(3)$, given at the identity by $\sigma$. By a result of Smale [16], this homogeneous space is contractible, and Theorem 5.1] can be applied to $\omega$.

\section{Appendix}

\subsection{Logarithmic derivative}

In this paragraph we collect some properties of the logarithmic derivative 8].

Given a manifold $M$ and a Lie group $G$ with Lie algebra $\mathfrak{g}$, the right logarithmic derivative of a function $h \in C^{\infty}(M, G)$ is $\delta^{r} h \in \Omega^{1}(M, \mathfrak{g})$ :

$$
\left(\delta^{r} h\right)\left(X_{x}\right):=\left(T_{x} h \cdot X_{x}\right) h(x)^{-1}, \forall X_{x} \in T_{x} M .
$$

Remark 8.1. A left logarithmic derivative $\delta^{l}$ is defined by similar formula $\left(\delta^{l} h\right)\left(X_{x}\right):=$ $h(x)^{-1}\left(T_{x} h . X_{x}\right)$. There is a relation between left and right logarithmic derivatives

$$
\delta^{r} h=\operatorname{Ad}(h) \delta^{l} h=-\delta^{l}\left(h^{-1}\right) .
$$

The right logarithmic derivative of a curve $h$ in the multiplicative group of positive real numbers is $\delta^{r} h\left(\partial_{t}\right)=\delta^{l} h\left(\partial_{t}\right)=(\log h)^{\prime}(t)$, the derivative of the logarithm of $h$.

Given a curve $h$ in $G$ we will identify the $\mathfrak{g}$-valued 1 -form $\delta^{r} h$ on $\mathbb{R}$ with the curve $i_{\partial_{t}} \delta^{r} h$ in $\mathfrak{g}$. When $\varphi_{t} \in \operatorname{Diff}(M)$ is a diffeotopy of the manifold $M$, then $\delta^{r} \varphi_{t}$ is the associated time dependent vector field on $M$, and $\delta^{l} \varphi_{t}=\varphi_{t}^{*} \delta^{r} \varphi_{t}$. For $\omega$ a differential form, we have $\frac{d}{d t} \varphi_{t}^{*} \omega=\varphi_{t}^{*} L_{\delta^{r} \varphi_{t}} \omega$. This is a particular case of the identity $\frac{d}{d t}\left(g_{t} \cdot v\right)=\delta^{r} g_{t} \cdot\left(g_{t} \cdot v\right)=g_{t} \cdot\left(\delta^{l} g_{t} \cdot v\right)$, for a smooth $G$-module $V$ and a curve $g_{t}$ in $G$.

Remark 8.2. Assume that $V$ is a Banach space. Given a closed form $\omega \in$ $\Omega^{p}(M, V)$, a path $\varphi_{t}$ of diffeomorphisms of $M$ and a path $u_{t}$ of linear isomorphisms of $V$, both starting at the identity, the following equivalences hold for every $t$ :

$$
\varphi_{t}^{*} \omega=u_{t} \cdot \omega \Leftrightarrow L_{\delta^{r} \varphi_{t}} \omega=\delta^{r} u_{t} \cdot \omega \Leftrightarrow L_{\delta^{l} \varphi_{t}} \omega=\delta^{l} u_{t} \cdot \omega .
$$

We show that $L_{\delta^{r} \varphi_{t}} \omega=\delta^{r} u_{t} \cdot \omega$ implies $\varphi_{t}^{*} \omega=u_{t} \cdot \omega$. The computation $\frac{d}{d t}\left(\varphi_{t}^{*} \omega-\right.$ $\left.u_{t} \cdot \omega\right)=\varphi_{t}^{*} L_{\delta^{r} \varphi_{t}} \omega-\delta^{r} u_{t} \cdot\left(u_{t} \cdot \omega\right)=\delta^{r} u_{t} \cdot\left(\varphi_{t}^{*} \omega-u_{t} \cdot \omega\right)$ shows that the curve $\omega_{t}=\varphi_{t}^{*} \omega-u_{t} \cdot \omega$ is a solution of the differential equation $\frac{d}{d t} \omega_{t}=\delta^{r} u_{t} \cdot \omega_{t}$ in $\Omega^{p}(M, V)$ with initial condition $\omega_{0}=0$. Evaluating at $p$ tangent vectors, we get a differential equation on the Banach space $V$, for which uniqueness of solutions is ensured. It follows that $\omega_{t}=0$, hence $\varphi_{t}^{*} \omega=u_{t} \cdot \omega$. 
Remark 8.3. The right logarithmic derivative satisfies the Leibniz rule

$$
\delta^{r}\left(h_{1} h_{2}\right)=\delta^{r} h_{1}+\operatorname{Ad}\left(h_{1}\right) \delta^{r} h_{2}
$$

for $h_{1}, h_{2} \in C^{\infty}(M, G)$. In other words $\delta^{r}$ is a group 1-cocycle on $C^{\infty}(M, G)$ with values in the $C^{\infty}(M, G)$-module $\Omega^{1}(M, \mathfrak{g})$ (for the adjoint action). The logarithmic derivative of a $G$-valued function $h$ on a connected manifold $M$ vanishes if and only if $h$ is constant. The Leibniz rule for the left logarithmic derivative is

$$
\delta^{l}\left(h_{1} h_{2}\right)=\delta^{l} h_{2}+\operatorname{Ad}\left(h_{2}^{-1}\right) \delta^{l} h_{1} .
$$

The left Maurer-Cartan form on a Lie group $G$ is the 1 -form

$$
\theta_{G}^{l}=\delta^{l}\left(\mathrm{id}_{G}\right) \in \Omega^{1}(G, \mathfrak{g}) .
$$

It satisfies $\theta_{G}^{l}\left(X_{g}\right)=g^{-1} \cdot X_{g}$ for $X_{g} \in T_{g} G$, and $\delta^{l} h=h^{*} \theta_{G}^{l}$ for any $h \in C^{\infty}(M, G)$. Both $\theta_{G}^{l}$ and $\delta^{l} h$ satisfy the right Maurer-Cartan equation

$$
d \theta_{G}^{l}+\frac{1}{2}\left[\theta_{G}^{l}, \theta_{G}^{l}\right]=0, \quad d \delta^{l} h+\frac{1}{2}\left[\delta^{l} h, \delta^{l} h\right]=0 .
$$

Given $\alpha \in \Omega^{1}(M, \mathfrak{g})$ which satisfies the right Maurer-Cartan equation $d \alpha+\frac{1}{2} \alpha \wedge \alpha=$ 0 and $U \subseteq M$ simply connected, there exists a smooth function $h: U \rightarrow G$ with $\delta^{l} h=\alpha$ on $U$. In the special case of a domain in $\mathbb{R}^{2}$, when $h(t, s) \in G$ is a smooth two parameter family, we get

$$
\frac{d}{d t} \eta-\frac{d}{d t} \xi=[\xi, \eta]
$$

where $\xi(t, s)=\left(\frac{d}{d t} h(t, s)\right) h(t, s)^{-1}$ and $\eta(t, s)=\left(\frac{d}{d s} h(t, s)\right) h(t, s)^{-1}$.

Proposition 8.4. Let $\theta \in \Omega^{1}(P, V)$ be a principal connection of the principal A-bundle $q: P \rightarrow M$ with $A=V / \Gamma$ and principal action $\rho$. Then for any $f \in C^{\infty}(P, A)$,

$$
\rho(f)^{*} \theta=\theta+\delta^{l}(f),
$$

where $\rho$ denotes the principal A-action.

Proof. Let $q^{-1}(U) \cong U \times A$ be a principal bundle chart with $q$ and $q_{A}$ the two projections on $U$ and $A$. The principal connection 1-form in this chart is

$$
\theta=q^{*} \alpha+q_{A}^{*} \theta_{A}^{l},
$$

where $\theta_{A}^{l}=\delta^{l}\left(1_{A}\right) \in \Omega^{1}(A, V)$ is the Maurer-Cartan form on $A$, and $\alpha \in \Omega^{1}(U, V)$ closed. The diffeomorphism $\rho(f)$ written in this chart is $\rho(f)=\left(q, m \circ\left(q_{A}, f\right)\right)$, with $m$ denoting the multiplication map in $A$. Now

$$
\rho(f)^{*} \theta=q^{*} \alpha+\left(m \circ\left(q_{A}, f\right)\right)^{*} \delta^{l}\left(1_{A}\right)=q^{*} \alpha+\delta^{l}\left(q_{A}\right)+\delta^{l}(f)=\theta+\delta^{l}(f),
$$

because by (40) $\delta^{l}\left(m \circ\left(h_{1}, h_{2}\right)\right)=\delta^{l} h_{1}+\delta^{l} h_{2}$ for $h_{1}, h_{2}: P \rightarrow A$. 


\section{$8.21-$ Cocycles}

We list in this subsection some properties of 1-cocycles on Lie algebras and Lie groups [10]. Let $G$ be a Lie group with Lie algebra $\mathfrak{g}$ and let $V$ be a smooth $G$ module. Then $V$ is a $\mathfrak{g}$-module and the pull-back action by the universal covering homomorphism $\tilde{G} \rightarrow G$ makes $V$ a $\tilde{G}$-module.

A $V$-valued group 1 -cocycle is a locally smooth map $a: G \rightarrow V$ with

$$
a\left(g g^{\prime}\right)=a(g)+g \cdot a\left(g^{\prime}\right)
$$

and a $V$-valued Lie algebra 1 -cocycle is a continuous linear map $\alpha: \mathfrak{g} \rightarrow V$ with

$$
\alpha\left(\left[X, X^{\prime}\right]\right)=X \cdot \alpha\left(X^{\prime}\right)-X^{\prime} \cdot \alpha(X) .
$$

There is a natural map $a \mapsto \alpha=d_{e} a$ from locally smooth group 1-cocycles on the Lie group $G$ to Lie algebra 1 -cocycles on its Lie algebra $\mathfrak{g}$.

Let $\alpha^{\text {eq }}$ be the equivariant $V$-valued 1-form on $G$ uniquely determined by the 1-cocycle $\alpha$ through $\alpha_{g}^{\mathrm{eq}}(g \cdot X)=g \cdot \alpha(X)$ for all $g \in G$ and $X \in \mathfrak{g}$. It is closed since $\alpha$ is a cocycle.

Proposition 8.5. If $a: G \rightarrow V$ is a group 1-cocycle integrating the Lie algebra 1 -cocycle $\alpha: \mathfrak{g} \rightarrow V$, then the following identities hold:

1. $d a=\alpha^{\mathrm{eq}} \in \Omega^{1}(G, V)$.

2. $\frac{d}{d t}\left(a\left(g_{t}\right)\right)=g_{t} \cdot \alpha\left(\delta^{l} g_{t}\right)=\alpha\left(\delta^{r} g_{t}\right)+\delta^{r} g_{t} \cdot a\left(g_{t}\right)$, for $g_{t}$ a path in $G$.

Proposition 8.6. There exists a unique group 1-cocycle on the universal covering group $\tilde{G}$ integrating the Lie algebra 1 -cocycle $\alpha: \mathfrak{g} \rightarrow V$

$$
\tilde{a}: \tilde{G} \rightarrow V, \quad \tilde{a}([g])=\int_{0}^{1} g_{t} \cdot \alpha\left(\delta^{l} g_{t}\right) d t
$$

where $\tilde{G}$ is identified with the group of homotopy classes of piecewise smooth paths $g_{t}$ in $G$ starting at the identity.

Proof. Because $d \alpha^{\mathrm{eq}}=0$, the map $\tilde{a}$ is well defined. The 1 -cocycle condition (43) for $\tilde{a}$ is easily verified noticing that the smooth path $g_{t} g_{t}^{\prime}, t \in[0,1]$ and the piecewise smooth path $h_{t}$, defined by $h_{t}=g_{2 t}$ if $t \leq \frac{1}{2}$ and $h_{t}=g_{1} g_{2 t-1}^{\prime}$ if $t \geq \frac{1}{2}$, are homotopic.

Remark 8.7. Let $\alpha: \mathfrak{g} \rightarrow V$ be a Lie algebra 1-cocycle. If the group $\Pi_{\alpha}$ of periods of the closed 1 -form $\alpha^{\mathrm{eq}}$ is discrete, then for any discrete subgroup $\Pi$ of $V$ containing $\Pi_{\alpha}$, the 1 -cocycle $\tilde{a}$ descends to a 1 -cocycle $a: G \rightarrow V / \Pi$.

From Proposition 8.5 and Remark 8.1 we deduce the following: 
Corollary 8.8. Let $a: G \rightarrow V / \Pi$ be a group 1-cocycle integrating the Lie algebra 1 -cocycle $\alpha: \mathfrak{g} \rightarrow V$. Then Ker $a$ is a subgroup of $G$ and Ker $\alpha$ is a Lie subalgebra of $\mathfrak{g}$ such that, for any smooth curve $g$ in $G$ starting at the identity, the following are equivalent: $g_{t} \in \operatorname{Ker} a \Leftrightarrow \delta^{l} g_{t} \in \operatorname{Ker} \alpha \Leftrightarrow \delta^{r} g_{t} \in \operatorname{Ker} \alpha$.

Remark 8.9. The results of this section about 1-cocycles hold also for diffeomorphism groups $G$ with Lie algebras $\mathfrak{g}$ of vector fields such that the following are equivalent: $g_{t} \in G \Leftrightarrow \delta^{l} g_{t} \in \mathfrak{g} \Leftrightarrow \delta^{r} g_{t} \in \mathfrak{g l}$.

\subsection{Obstructions to integrability}

According to the general theory developed in [9] and [10], there are two obstructions for the integration of a Lie algebra cocycle $\sigma$ on $\mathfrak{g}$ with values in a $G$-module $V$ to a Lie group extension of $G$ by a quotient group of $V$ : the period map and the flux homomorphism.

Let $\sigma^{\text {eq }}$ be the closed equivariant $V$-valued 2-form on $G$ uniquely determined by the 2-cocycle $\sigma$. The period map is the group homomorphism

$$
\operatorname{per}_{\sigma}: \pi_{2}(G) \rightarrow V^{G}, \quad \operatorname{per}_{\sigma}([c])=\int_{S^{2}} c^{*} \sigma^{\mathrm{eq}} \quad \text { for } \quad c \in C^{\infty}\left(S^{2}, G\right)
$$

Its image $\Pi_{\sigma}$ is called the period group of $\sigma$.

The flux homomorphism $F_{\sigma}: \pi_{1}(G) \rightarrow H^{1}(\mathfrak{g}, V),[\gamma] \mapsto\left[I_{\gamma}^{\sigma}\right]$, assigns to each piecewise smooth loop $\gamma$ in $G$ based at the identity, the cohomology class of the 1 -cocycle

$$
I_{\gamma}^{\sigma}: \mathfrak{g} \rightarrow V, \quad I_{\gamma}^{\sigma}(X)=-\int_{\gamma} i_{X^{r}} \sigma^{\mathrm{eq}}
$$

Theorem 8.10. For a Lie algebra $V$-valued 2-cocycle $\sigma$ on $\mathfrak{g}$ with discrete period group $\Pi_{\sigma}$ and vanishing flux homomorphism $F_{\sigma}$, the abelian Lie algebra extension $0 \rightarrow V \rightarrow \hat{\mathfrak{g}} \rightarrow \mathfrak{g} \rightarrow 0$ defined by $\sigma$ integrates to an abelian Lie group extension

$$
1 \rightarrow V / \Pi_{\sigma} \rightarrow \hat{G} \rightarrow G \rightarrow 1 .
$$

Acknowledgements. The author thanks Karl-Hermann Neeb for many very useful comments and suggestions. The valuable remarks and corrections due to the anonymous referee, especially those concerning Theorem 5.2, are gratefully acknowledged.

\section{References}

[1] Banyaga, A.: Sur la structure du groupe des diffeomorphismes qui preservent une forme symplectique. Comment. Math. Helv. 53, 174-227 (1978) 
[2] Billig, Y.: Abelian extensions of the group of diffeomorphisms of a torus. Lett. Math. Phys. 64, 155-169 (2003)

[3] Brylinski, J.-L.: Loop spaces, characteristic classes and geometric quantization, Progress in Math. 107, Birkhäuser (1993)

[4] Haller, S., Vizman, C.: Non-linear Grassmannians as coadjoint orbits. Math. Ann. 329, 771-785 (2004)

[5] Ismagilov, R.S.: Representations of Infinite-Dimensional Groups. AMS Translations of Mathematical Monographs 152, Amer. Math. Soc. (1996)

[6] Ismagilov, R.S., Losik, M., Michor, P.W.: A 2-cocycle on a group of symplectomorphisms. Moscow Math. J. 6, 307-315 (2006)

[7] Kostant, B.: Quantization and unitary representations. pp. 87-208, Lecture Notes in Math. 170, Springer (1970)

[8] Kriegl, A., Michor, P.W.: The Convenient Setting of Global Analysis. Mathematical Surveys and Monographs 53, Amer. Math. Soc. (1997)

[9] Neeb, K.-H.: Central extensions of infinite-dimensional Lie groups, Ann. Inst. Fourier 52, 1365-1442 (2002)

[10] Neeb, K.-H.: Abelian extensions of infinite-dimensional Lie groups. Travaux Math. XV, 69-194 (2004)

[11] Neeb, K.-H., Vizman, C.: Flux homomorphisms and principal bundles over infinite dimensional manifolds. Monatsh. Math. 139, 309-333 (2003)

[12] Ono, K.: Floer-Novikov cohomology and the flux conjecture. Geom. Funct. Anal. 16, 981-1020 (2006)

[13] Ovsienko, V.Y., Roger, C.: Generalizations of Virasoro group and Virasoro algebra through extensions by modules of tensor densities on $S^{1}$. Indag. Math. N.S. 9, 277-288 (1998)

[14] Ratiu, T., Schmid, R.: The differentiable structure of three remarkable diffeomorphism groups. Math. Z. 177, 81-100 (1981)

[15] Roger, C.: The group of volume preserving diffeomorphisms and the Lie algebra of unimodular vector fields: survey of some classical and not-so-classical results. In: Twenty years of Bialowieza: A mathematical antology. Aspects of differential geometric methods in physics, pp. 79-98, World Scientific Monograph Series in Math. 8 (2005)

[16] Smale, S.: Diffeomorphisms of the two sphere. Proc. Amer. Math. Soc. 10, 621-626 (1959) 
[17] Souriau, J.-M.: Structure des systemes dynamiques. Dunod, Paris (1970)

[18] Weinstein, A., Zambon, M.: Variations on prequantization. Travaux Math. XVI, 187-219 (2005) 